\title{
How heavy are the tails of a stationary HARCH(k) process? A study of the moments *广
}

\author{
PAUL EMBRECHTS \\ ETH ZENTRUM \\ MiCHEL M. DACOROGNA \\ OLSEN \& ASSOCIATES
}

\author{
GENNADY SAMORODNITSKY \\ CORNELL UNIVERSITY \\ ULRICH A. MÜLLER \\ OLSEN \& ASSOCIATES
}

September 5, 1996

\begin{abstract}
Probabilistic properties of HARCH(k) processes, as special stochastic volatility models, are investigated. We present necessary and sufficient conditions for existence of a stationary version of a HARCH( $k)$ process with finite $(2 m)$ th moments, $m \geq 1$. Our approach is based on the general Markov chain techniques of (Meyn and Tweedie, 1990). The conditions are explicit in the case of second moments, and also in the case of 4th moments of the HARCH(2) process. We also deduce explicit necessary and explicit sufficient conditions for higher order moments of general HARCH(k) models. We start by studying the HARCH(2) process (in which case our results are the most explicit) and then generalize the results to a general $H A R C H(k)$ process.
\end{abstract}

\section{Introduction}

In (Müller et al., 1995), the Heterogeneous Auto-Regressive Conditional Heteroskedastic process $(\mathrm{HARCH})$ has been introduced. This $\mathrm{HARCH}$ process has been developed as an improvement of traditional ARCH-type models in order to describe the behavior of financial time series, including some newly detected properties such as the long memory of volatility and the asymmetry between volatilities with different degrees of resolution in time.

The HARCH process is useful in practice only if its basic properties are known. In section 2, we give some motivation for using $\mathrm{HARCH}$ and determining its stationarity and moment conditions.

The HARCH process is a random recursion, whose equations are presented in section 3 . In order to understand how heavy are the tails of a stationary $\mathrm{HARCH}(\mathrm{k})$ process, we study its moments. In section 4 , we start by presenting in more details how a necessary condition for the existence of the

\footnotetext{
${ }^{*}$ AMS 1991 subject classification. Primary 60K30, 90A09. Secondary 60H25

${ }^{\dagger}$ Keywords and phrases: HARCH processes, heavy tails, Markov chains, heteroscedasticity, Harris recurrence, random recusrion, stochastic volatility.
} 


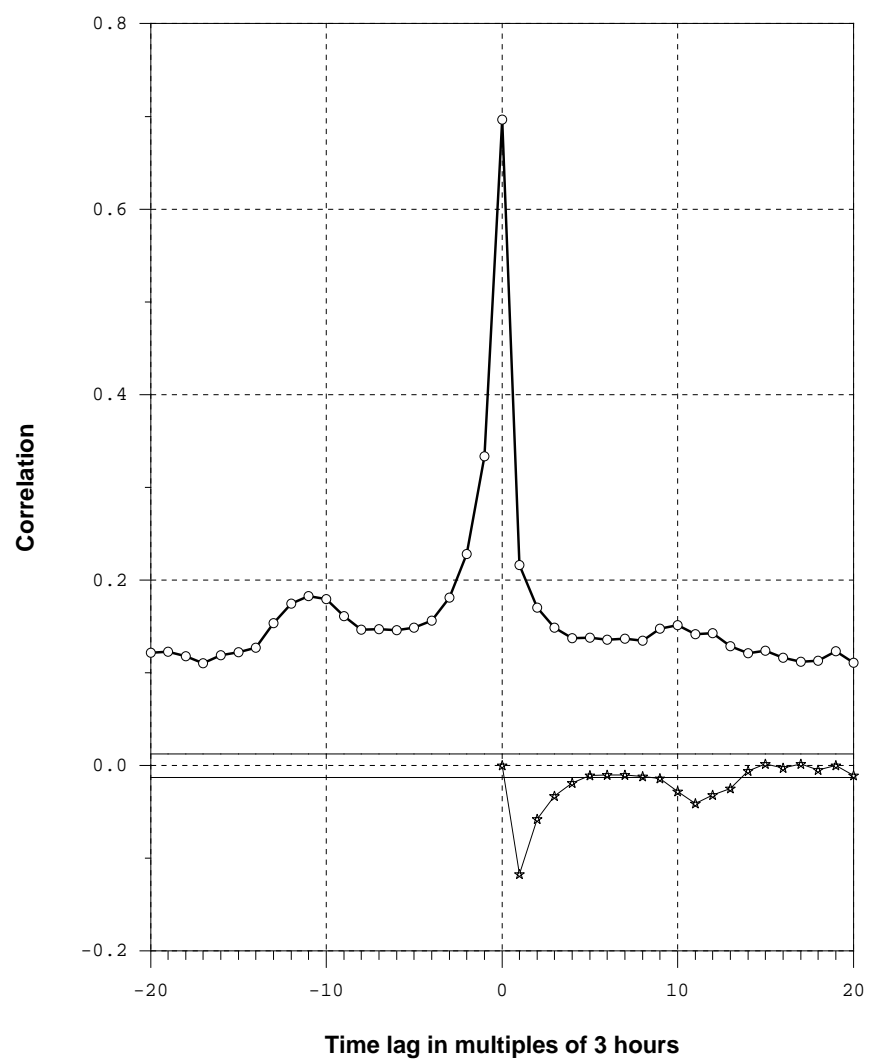

Lead-lag correlation of fine and coarse volatilities of a USD/DEM time series with a half-hourly grid in $\vartheta$-time, as defined in (Dacorogna et al., 1993). The fine volatility is defined as the mean absolute half-hourly price change within 3 hours ( $\vartheta$-time); the coarse volatility is the absolute price change over a whole 3 hour interval ( $\vartheta$-time). The thin curve indicates the asymmetry: the difference between correlations at positive and corresponding negative lags. Sampling period: 8 years, from 1 Jan 1987 00:00 to 1 Jan 1995 00:00 (GMT). The confidence limits represent the $95 \%$ confidence interval of a Gaussian random walk.

Figure 1: Asymmetric lead-lag correlation of fine and coarse volatilities of a half-hourly USD/DEM series

2nd moment can be derived and to show using a Markov chain approach that the necessary condition presented in (Müller et al., 1995) is also sufficient for the existence of a stationary HARCH(k) process with a finite second moment. This is done first for the $\mathrm{HARCH}(2)$ process and then generalized to $\mathrm{HARCH}(\mathrm{k})$. As a rule, we first analyze the $\mathrm{HARCH}(2)$ process, and then consider the general $\mathrm{HARCH}(\mathrm{k})$ process. The reason for this is two-fold. First of all, the results are often most explicit for the HARCH(2) model. Second, the arguments and main ideas are the easiest to follow in this case.

With the above in mind, we next present the derivation of an explicit necessary and sufficient condition for the existence of the 4th moment of a HARCH(2) model. The following step is to derive a general theorem giving necessary and sufficient conditions for the existence of a stationary $\mathrm{HARCH}(2)$ process with finite $(2 m)$ th moments. We are then able to derive an explicit necessary condition for a finite $(2 m)$ th moment of an $\mathrm{HARCH}(2)$ and derive an explicit sufficient condition for the existence of the $(2 m)$ th moment.

The second part of the paper contains a generalization to $\mathrm{HARCH}(\mathrm{k})$ models. As mentioned above, we start by proving an explicit necessary and sufficient condition for a finite 2 nd moment. We further give a general theorem for stationarity with finite moments, where we prove that the condition is both necessary and sufficient. The condition is in terms of existence of a nonnegative solution of a certain system of linear equations. For higher order moments of HARCH(k) models we give an explicit necessary condition and an explicit sufficient condition for a finite $(2 m)$ th moment.

\section{Motivation}




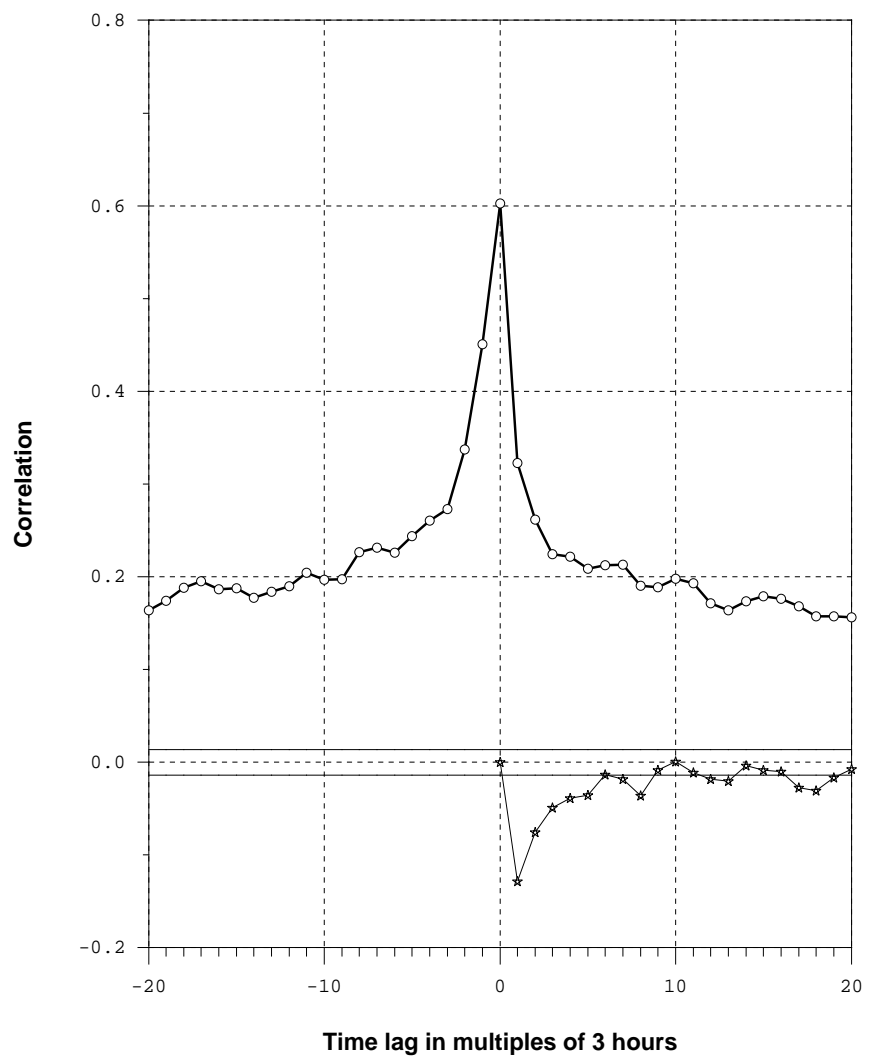

Lead-lag correlation of fine and coarse volatilities of a synthetic HARCH time series, fitted to a USD/DEM series on a half-hourly time grid in $\vartheta$-time. The fine volatility is defined as the mean absolute half-hourly price change in an interval of 3 hours; the coarse volatility is the absolute price change over the whole 3hour interval. The thin curve indicates the asymmetry: the difference between correlations at positive and corresponding negative lags. Sampling period: 7 years. The confidence limits represent the $95 \%$ confidence interval of a Gaussian random walk.

Figure 2: Asymmetric lead-lag correlation of fine and coarse volatilities of a HARCH series

The HARCH process has been developed for describing the behavior of financial time series, with price quotes from the foreign exchange market being the best-studied example; see (Müller et al., 1995). Financial time series exhibit clusters of high and low volatility, i. e. autoregressive conditional heteroskedasticity.

Additional facts have been found in recent empirical research of high-frequency data in finance: (1) a long memory in the volatility (positive autocorrelation of absolute price changes declining slower than exponentially) and (2) asymmetry between volatilities observed with different time resolutions. The HARCH process precisely reproduces these empirical facts.

The asymmetry between different volatilities deserves some special attention here as it is a quite newly detected effect. Figure 1 shows that the lead-lag correlation between a high-resolution volatility and another volatility measured with lower resolution in time is asymmetric: low-resolution (coarse) volatility predicts high-resolution (fine) volatility better than the other way around. In Figure 1, the case of the US Dollar expressed in German Marks (USD/DEM) is shown, but the effect is found also for all other foreign exchange rates we studied.

Figure 2 shows the case of a time series synthetically generated from a HARCH process whose 8 parameters were fitted on a long USD/DEM sample. This synthetic data exhibits the same asymmetric behavior of lead-lag correlation as the empirical USD/DEM data in Figure 1, whereas the other ARCH-type processes lead to symmetric lead-lag correlograms. More details on this study can be found in (Müller et al., 1995).

The correct reproduction of many empirical properties makes $\mathrm{HARCH}$ an attractive choice for modeling the behavior of financial time series. In order to use a HARCH model in practice, however, 

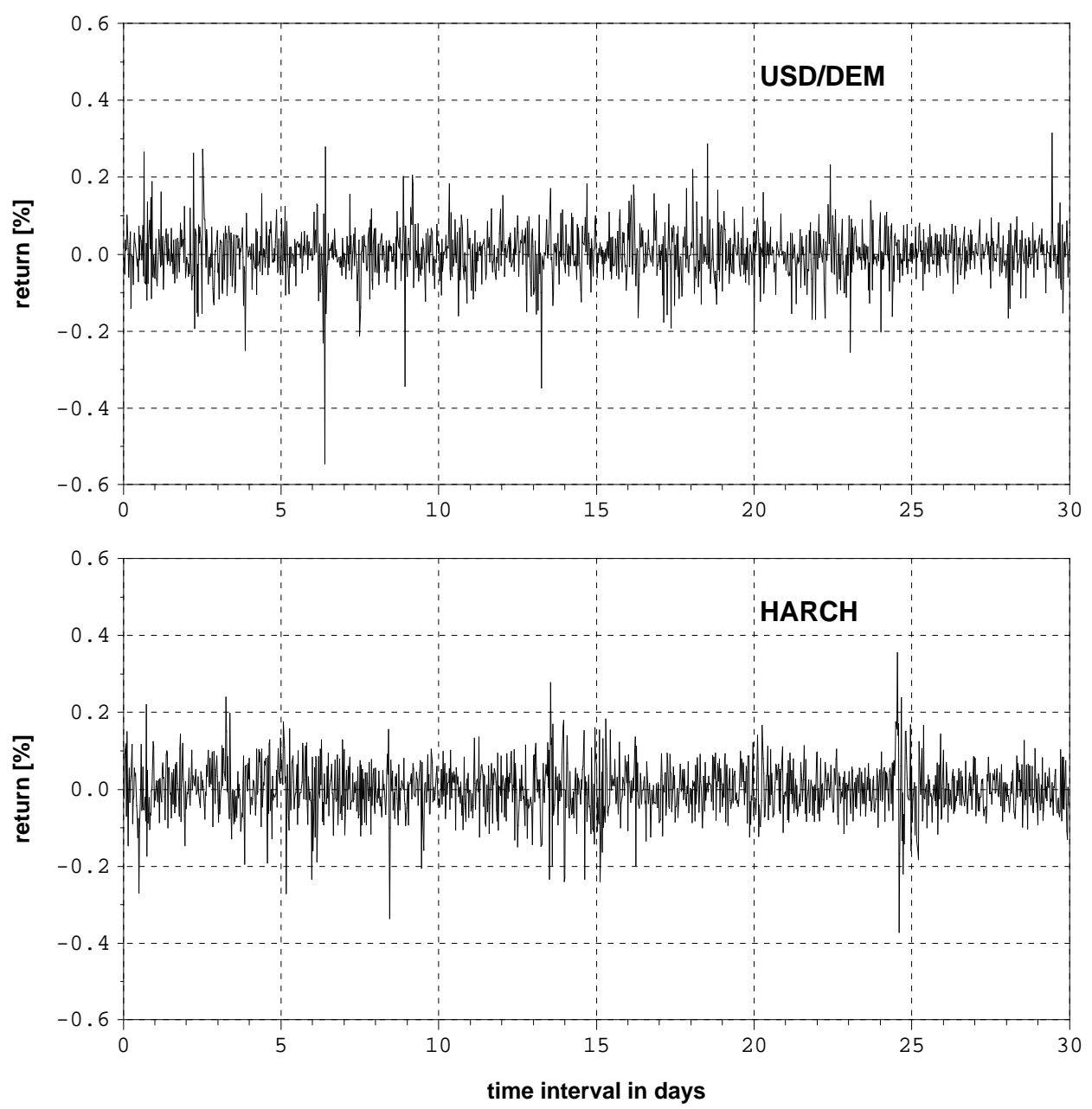

Figure 3: A comparison between 1 month of 30 minutes (in business time scale) returns of the USD/DEM foreign exchange rate and a Monte-Carlo realization of a $\mathrm{HARCH}$ process fitted to the same rate over 30 minute time intervals.

some basic theoretical properties of HARCH should be known, among these certainly the conditions for the stationarity and the existence of finite higher moments of the unconditional distribution function are crucial.

There is yet another motivation for studying the moment conditions. Financial risk analysis is based upon assumptions on the probability of extreme price movements. Extreme events in the tails of the distribution function are strongly related to the existence of the higher moments. If the $(2 \mathrm{~m})$ th moment of a distribution is finite and the $(2(m+1))$ th moment is not, the tail index of the distribution, if this distribution has a regularly varying tail, must lie between $2 m$ and $2(m+1)$. The knowledge of the moment conditions might help to use HARCH in financial risk management.

\section{The HARCH process}

The HARCH(k) process equations as first presented in (Müller et al., 1995) are: specify $r_{0}, \ldots, r_{k-1}$ and

$$
r_{n}=\sigma_{n} \varepsilon_{n}, n=0,1,2, \ldots
$$




$$
\begin{aligned}
& \sigma_{n}^{2}=c_{0}+\sum_{j=1}^{k} c_{j}\left(\sum_{i=1}^{j} r_{n-i}\right)^{2}, \\
& c_{0}>0, c_{k}>0, c_{j} \geq 0 \text { for } j=1 \ldots k-1,
\end{aligned}
$$

where $r_{n}$ is the return of a financial asset time series as defined in (Guillaume et al., 1994). The $\epsilon_{n}{ }^{\prime}$ s are independent identically distributed (iid) symmetric random variables, whose distribution is assumed to have a non-zero absolutely continuous component, which has a positive density on a Borel set with a non-empty interior. Typical examples are a normal distribution $N\left(0, \sigma^{2}\right)$ or a Student-t distribution with zero expectation. Denote $a_{m}=\mathrm{E}\left(|\epsilon|^{2 m}\right), m \geq 1$. For example, if the $\epsilon_{n}{ }^{\prime} \mathrm{s}$ have the standard normal distribution, then it is well known that $a_{m}=\prod_{j=1}^{m}(2 j-1), m \geq 1$. We will always assume that $\epsilon_{n}$ has a finite absolute moment of at least the same order as we want $r_{n}$ to have. (That is, if we are discussing, say, conditions for existence of the finite 6 th moment of $r_{n}$, we will assume that $E\left(\left|\epsilon_{n}\right|^{6}\right)<\infty$.) In (Müller et al., 1995) the moment conditions deduced in this paper were first announced.

In Figure 3, we present a realization of a HARCH process fitted to the returns of the USD/DEM foreign exchange rate measured over 30 minute time intervals. The figure displays 1 month of 30 minutes returns for the real FX rate (measured on the business time scale described in (Dacorogna et al., 1993)) and a Monte-Carlo realization of the fitted HARCH process. Both graphs present fat-tails and heteroskedasticity although not exactly at the same place since the realization of the $\mathrm{HARCH}$ process is drawn with a random number generator for the $\varepsilon_{n}$. The similarity in behavior between the two curves is striking and is confirmed by other statistical analysis see (Müller et al., 1995).

\section{Condition for stationarity with a finite 2nd moment for HARCH(2)}

\subsection{Necessity}

Let us first recall the way to get to the condition for the existence of a stationary distribution with a finite 2 nd moment for a $\mathrm{HARCH}(2)$ process. The equation of such a process is:

$$
\begin{aligned}
r_{n} & =\sigma_{n} \varepsilon_{n}, n=0,1,2, \ldots, \\
\sigma_{n}^{2} & =c_{0}+c_{1} r_{n-1}^{2}+c_{2}\left(r_{n-1}+r_{n-2}\right)^{2} .
\end{aligned}
$$

The scaled returns $\varepsilon_{n}$ satisfy the assumptions of the previous section. If $r_{n}$ is stationary with a finite 2nd moment, then

$$
\mathrm{E}\left(r_{n}^{2}\right)=\mathrm{E}\left(r_{n-i}^{2}\right), \quad i \geq 1
$$

Recall that $a_{1}=\mathrm{E}\left(\epsilon_{n}\right)^{2}$. If we now use (4.1), we can write:

$$
\mathrm{E}\left(r_{n}^{2}\right)=a_{1} \mathrm{E}\left(\sigma_{n}^{2}\right)=a_{1}\left(c_{0}+\left(c_{1}+c_{2}\right) \mathrm{E}\left(r_{n-1}^{2}\right)+c_{2} \mathrm{E}\left(r_{n-2}^{2}\right)+2 c_{2} \mathrm{E}\left(r_{n-1} r_{n-2}\right)\right) .
$$

Since we know that $\varepsilon_{n}$ is iid with mean zero, the expectation of the cross product is zero and using (4.2) we obtain

$$
\mathrm{E}\left(r_{n}^{2}\right)=\frac{c_{0}}{\frac{1}{a_{1}}-\left[\left(c_{1}+c_{2}\right)+c_{2}\right]} .
$$


The reason why we write the denominator this way will become clear later. A necessary condition for stationarity with a finite 2 nd moment for a $\mathrm{HARCH}(2)$ process then becomes

$$
\left(c_{1}+c_{2}\right)+c_{2}<\frac{1}{a_{1}}
$$

which is equivalent to (3.6) in (Müller et al., 1995) when $k=2$ (see condition (6.10) for the reason for writing the left hand side above in this form).

\subsection{Sufficiency of the condition $\left(c_{1}+c_{2}\right)+c_{2}<1 / a_{1}$}

The derivation of the expression of $\mathrm{E}\left(r_{n}^{2}\right)$ in (4.4) as a function of the coefficients $c_{1}$ and $c_{2}$ is not sufficient to prove the existence of a stationary version of the $\mathrm{HARCH}(2)$ process with finite second moments. In order to do this, we need to reformulate the problem in terms of a Markov chain. We do not follow here the path chosen by (Engle, 1982) and (Bollerslev, 1986) because of the cross term $r_{n-1} r_{n-2}$ which makes the matrix formulation of the problem difficult.

With the HARCH(2) process $\left(r_{n}\right)$ we can associate a two-dimensional Markov chain

$$
X_{n}=\left(r_{n-1}, r_{n}\right), n \geq 1 \text {. }
$$

The various properties of $\left(r_{n}\right)$ can now readily be derived through standard results on Markov chains with state space $\mathbb{R}^{2}$. We base our analysis strongly on an excellent book by Mein and Tweedie (1990), where one can find many results that give sufficiency of various recurrence conditions and existence of stationary distributions with finite moments. The latter, in our language, means existence of a stationary solution to (4.1) with appropriate finite moments.

Let us summarize the main results. Let $\left\{X_{n}, n \geq 0\right\}$ be a Markov chain, with values in an Euclidian space. For such Markov chains the key notion is that of an irreducible T-chain. Because of the conditions on the density of the noise variables $\varepsilon_{n}$ 's, the latter is equivalent to showing the follwing continuity property:

(C) The conditional distribution of $X_{n}$ given $X_{n-1}=y_{k}$ converges weakly to that of $X_{n}$ given $X_{n-1}=y$ if $y_{k} \rightarrow y$.

This result is part of Theorem 6.0.1 in (Meyn and Tweedie, 1990). See also the relevant definitions on irredicible $T$-chains (pages 87,127 ) and the result that for a $T$-chain, every compact set is petite (page 121).

There are three levels of theorems in (Meyn and Tweedie, 1990) that will bring us to the desired result:

- Level 1: Suppose that there exists $V \geq 0$ with $V \not \equiv 0$ and a compact set $C$ such that

$$
V\left(X_{n}\right)-P V\left(X_{n}\right) \geq 0 \text { for } X_{n} \notin C,
$$

where $P$ expresses the expectation value at the following step of the Markov chain, i.e. $P V(x)=$ $\mathrm{E}\left(V\left(X_{n}\right) \mid X_{n-1}=x\right)$. Then there exists an invariant measure and the Markov chain is Harris recurrent.

This is essentially Theorem 12.3.3 of (Meyn and Tweedie, 1990). 
- Level 2: Suppose that there exists $V \geq 0$ and $V \not \equiv 0$ and a compact set $C$ such that

$$
V\left(X_{n}\right)-P V\left(X_{n}\right) \geq-b 1_{C}\left(X_{n}\right)+1
$$

where $1_{C}$ is the indicator function of the set $C$ ( 1 if in $C, 0$ if outside), and $\mathrm{b}$ a finite positive number. Then there exists a unique stationary distribution $\pi$ and $\left(X_{n}\right)$ is positive Harris recurrent.

This is essentially Theorem 11.3.4 or 13.0.1(iv) of (Meyn and Tweedie, 1990). Level 2 gives a sufficient condition for the stationarity of the Markov chain.

- Level 3: Suppose that $X_{n}$ is positive Harris recurrent and there exist non-negative measurable functions $f, V$ so that

$$
V\left(X_{n}\right)-P V\left(X_{n}\right) \geq f\left(X_{n}\right)-b
$$

with a finite $b \geq 0$. Then the $\pi$-expectation of $f, \pi f=\int f(x) \pi(d x)$, is finite, specifically: $\pi f \leq b<\infty$.

This is essentially Theorem 14.3.7 of (Meyn and Tweedie, 1990). Level 3 gives a sufficient condition for the existence of the appropriate moments of the stationary distribution.

If all three levels hold, we have established a sufficient condition for stationarity (level 2) and existence of moments (level 3).

To prove the ergodicity and existence of moments of $\mathrm{HARCH}(2)$, or, indeed, for any $\mathrm{HARCH}(\mathrm{k})$, we thus need to prove the weak continuity (C) of the distribution of $X_{n}$ given $X_{n-1}=y$ in $y$, and then to find a condition on the coefficients of the model so that levels 2 and 3 hold. The main idea behind the above approach is to prove that the Markov chain tends to drfift back to a neighbourhood of its initial position from any position far out.

\subsubsection{Continuity condition (C) on $X$}

We consider a Markov chain $\left(X_{n}\right)$ as defined in (4.6). To prove that it is a T-chain, we need to examine the continuity condition (C). Because of (4.1) and (4.6) every new state is described by analytic functions of the previous state and a new random variable. Thus the weak continuity of the Markov chain holds by the continuous mapping theorem (see (Billingsley, 1968), Theorem 5.1 page 30).

\subsubsection{Drift from the tails to the center}

We now turn to show that our Markov chain eventually tends to drift back to the center if the coefficients $c_{1}, c_{2}$ satisfy condition (4.5), $c_{1}+2 c_{2}<1 / a_{1}$. We start by choosing a number $0<\alpha<1$ such that

(i) $\alpha>c_{2} a_{1}$,

(ii) $c_{1}+c_{2}+\alpha / a_{1}<1 / a_{1}$.

We then define a function $V$ as:

$$
V(x, y) \equiv \alpha x^{2}+y^{2}+2 c_{2} a_{1} x y
$$


where, because of condition (i) above, $V \geq 0$. Indeed the discriminant satisfies

$$
c_{2}^{2} a_{1}^{2}-\alpha<c_{2} a_{1}-\alpha<0
$$

By inserting (4.10) and (4.1),

$$
\begin{gathered}
V\left(r_{n-1}, r_{n}\right)-P V\left(r_{n-1}, r_{n}\right)=\alpha r_{n-1}^{2}+2 c_{2} a_{1} r_{n-1} r_{n}+r_{n}^{2}- \\
-E\left[\alpha r_{n}^{2}+2 c_{2} a_{1} r_{n} \varepsilon_{n+1}\left(c_{0}+c_{1} r_{n}^{2}+c_{2}\left(r_{n}+r_{n-1}\right)^{2}\right)^{1 / 2}+\right. \\
\left.+\varepsilon_{n+1}^{2}\left(c_{0}+c_{1} r_{n}^{2}+c_{2}\left(r_{n}+r_{n-1}\right)^{2}\right)\right] .
\end{gathered}
$$

Using $\mathrm{E}\left(\varepsilon_{n}\right)=0$ and $\mathrm{E}\left(\varepsilon_{n}^{2}\right)=a_{1}$, we have

$$
V\left(r_{n-1}, r_{n}\right)-P V\left(r_{n-1}, r_{n}\right)=\left(\alpha-c_{2} a_{1}\right) r_{n-1}^{2}+\left(1-\alpha-\left(c_{1}+c_{2}\right) a_{1}\right) r_{n}^{2}-c_{0} a_{1} .
$$

The two coefficients $\alpha-c_{2} a_{1}$ and $1-\alpha-\left(c_{1}+c_{2}\right) a_{1}$ are positive, and the last expression can be made as large as we wish if $\left(r_{n-1}, r_{n}\right)$ is outside of a compact set.

Now, under condition (4.5), we have found a function $V$ that satisfies the requirements of level 2 and also 3 with $f\left(x_{1}, x_{2}\right)=\theta\left(x_{1}^{2}+x_{2}^{2}\right)$, with $0<\theta<\left(\alpha-c_{2} a_{1}\right) \wedge\left(1-\alpha-\left(c_{1}+c_{2}\right)\right)$. Thus the stationarity and the existence of the second moment are proven if (4.5) holds.

\section{Existence of the 4th moment of a HARCH(2)}

\subsection{A necessary condition}

Since $r_{n}=\sigma_{n} \varepsilon_{n}$, we deduce that:

$$
\mathrm{E}\left(r_{n}^{4}\right)=\mathrm{E}\left(\sigma_{n}^{4}\right) \mathrm{E}\left(\varepsilon_{n}^{4}\right)=a_{2} \mathrm{E}\left(\sigma_{n}^{4}\right)
$$

Therefore,

$$
\mathrm{E}\left(\sigma_{n}^{4}\right)=\frac{1}{a_{2}} \mathrm{E}\left(r_{n}^{4}\right)
$$

With the help of (4.1), we obtain:

$$
\begin{aligned}
\mathrm{E}\left(\sigma_{n}^{4}\right)= & \mathrm{E}\left(\left[c_{0}+c_{1} r_{n-1}^{2}+c_{2}\left(r_{n-1}+r_{n-2}\right)^{2}\right]^{2}\right) \\
= & c_{0}^{2}+2 c_{0}\left(c_{1}+c_{2}\right) \mathrm{E}\left(r_{n-1}^{2}\right)+\left(c_{1}+c_{2}\right)^{2} \mathrm{E}\left(r_{n-1}^{4}\right)+2 c_{0} c_{2} \mathrm{E}\left(r_{n-2}^{2}\right)+ \\
& +2 c_{2}\left(c_{1}+3 c_{2}\right) \mathrm{E}\left(r_{n-1}^{2} r_{n-2}^{2}\right)+c_{2}^{2} \mathrm{E}\left(r_{n-2}^{4}\right),
\end{aligned}
$$

where we have used

$$
\mathrm{E}\left(r_{n-1} r_{n-2}\right)=\mathrm{E}\left(r_{n-1}^{3} r_{n-2}\right)=\mathrm{E}\left(r_{n-1} r_{n-2}^{3}\right)=0
$$


deduced from the iid nature of $\varepsilon_{n}{ }^{\prime}$ s and their symmetric distribution. For ease of notation, we define:

$$
L=\mathrm{E}\left(r_{n}^{2}\right), \quad M_{0}=\mathrm{E}\left(r_{n}^{4}\right) \text { and } \quad M_{2}=\mathrm{E}\left(r_{n-1}^{2} r_{n-2}^{2}\right)
$$

which do not depend on $n$ if the process is stationary. Rewrite (5.3) as

$$
\frac{1}{a_{2}} M_{0}=c_{0}^{2}+2 c_{0}\left(c_{1}+2 c_{2}\right) L+\left(c_{2}^{2}+\left(c_{1}+c_{2}\right)^{2}\right) M_{0}+2 c_{2}\left(c_{1}+3 c_{2}\right) M_{2}
$$

and compute, in a similar way, an expression for $M_{2}$ :

$$
\begin{aligned}
M_{2} & =\mathrm{E}\left(r_{n}^{2} r_{n-1}^{2}\right)=\mathrm{E}\left(\sigma_{n}^{2} r_{n-1}^{2}\right) E\left(\varepsilon_{n}^{2}\right)=a_{1} \mathrm{E}\left(\sigma_{n}^{2} r_{n-1}^{2}\right) \\
& =a_{1} \mathrm{E}\left(r_{n-1}^{2}\left[c_{0}+c_{1} r_{n-1}^{2}+c_{2}\left(r_{n-1}+r_{n-2}\right)^{2}\right]\right) \\
& =a_{1}\left(c_{0} L+\left(c_{1}+c_{2}\right) M_{0}+c_{2} M_{2}\right) .
\end{aligned}
$$

We are now left with a system of two equations with two variables $M_{0}$ and $M_{2}$ (the quantity $L$ is known from the stationarity condition (4.4)):

$$
\left\{\begin{array}{cl}
{\left[1-a_{2}\left(c_{2}^{2}+\left(c_{1}+c_{2}\right)^{2}\right)\right] M_{0}-2 a_{2} c_{2}\left(c_{1}+3 c_{2}\right) M_{2}} & =a_{2} c_{0}^{2}+2 a_{2} c_{0}\left(c_{1}+2 c_{2}\right) L \\
-\left(c_{1}+c_{2}\right) M_{0}+\left(\frac{1}{a_{1}}-c_{2}\right) M_{2} & =c_{0} L .
\end{array},\right.
$$

This linear system can be solved by standard methods, yielding

$$
M_{0}=\frac{a_{2} c_{0}^{2}\left(1-a-1 c_{2}\right)+2 a_{2} c_{0}\left(c_{1}+2 c_{2}+c_{2}^{2}\right) L}{\left(1-a_{1} c_{2}\right)-a_{2}\left[c_{2}^{2}+\left(c_{1}+c_{2}\right)^{2}\right]-a_{1} a_{2} c_{2}\left(c_{1}^{2}+6 c_{1} c_{2}+4 c_{2}^{2}\right)} .
$$

Because of the condition (4.5), the numerator is positive and hence a necessary condition for the existence of a stationary $\mathrm{HARCH}(2)$ process with finite 4 th moment becomes

$$
1-a_{2}\left[c_{2}^{2}+\left(c_{1}+c_{2}\right)^{2}\right]-a_{1} c_{2}\left[1+a_{2}\left(c_{1}^{2}+6 c_{1} c_{2}+4 c_{2}^{2}\right)\right]>0 .
$$

This condition is more stringent than (4.5). The solution for $M_{2}$ is:

$$
M_{2}=\frac{a_{1} a_{2} c_{0}^{2}\left(c_{1}+c_{2}\right)}{D}+\frac{2 a_{1} a_{2} c_{0}\left(c_{1}+c_{2}\right)\left(c_{1}+2 c_{2}+a_{1} c_{2}^{2}\right)}{\left(1-c_{2}\right) D} L+\frac{a_{1} c_{0}}{1-c_{2}} L
$$

where $D$ is the denominator of the right hand side of (5.8).

An easy check of this condition is to compare it to the condition for an ARCH(1) process. We know that $\mathrm{HARCH}(2)$ becomes $\mathrm{ARCH}(1)$ if $c_{2}=0$. On page 992 of (Engle, 1982), the condition for the existence of the $2 m$ th moment is given. Assume that the $\epsilon_{n}$ 's have the standard normal distribution. In that case, translated into our notation that condition becomes:

$$
c_{1}^{m} \prod_{j=1}^{m}(2 j-1)<1
$$




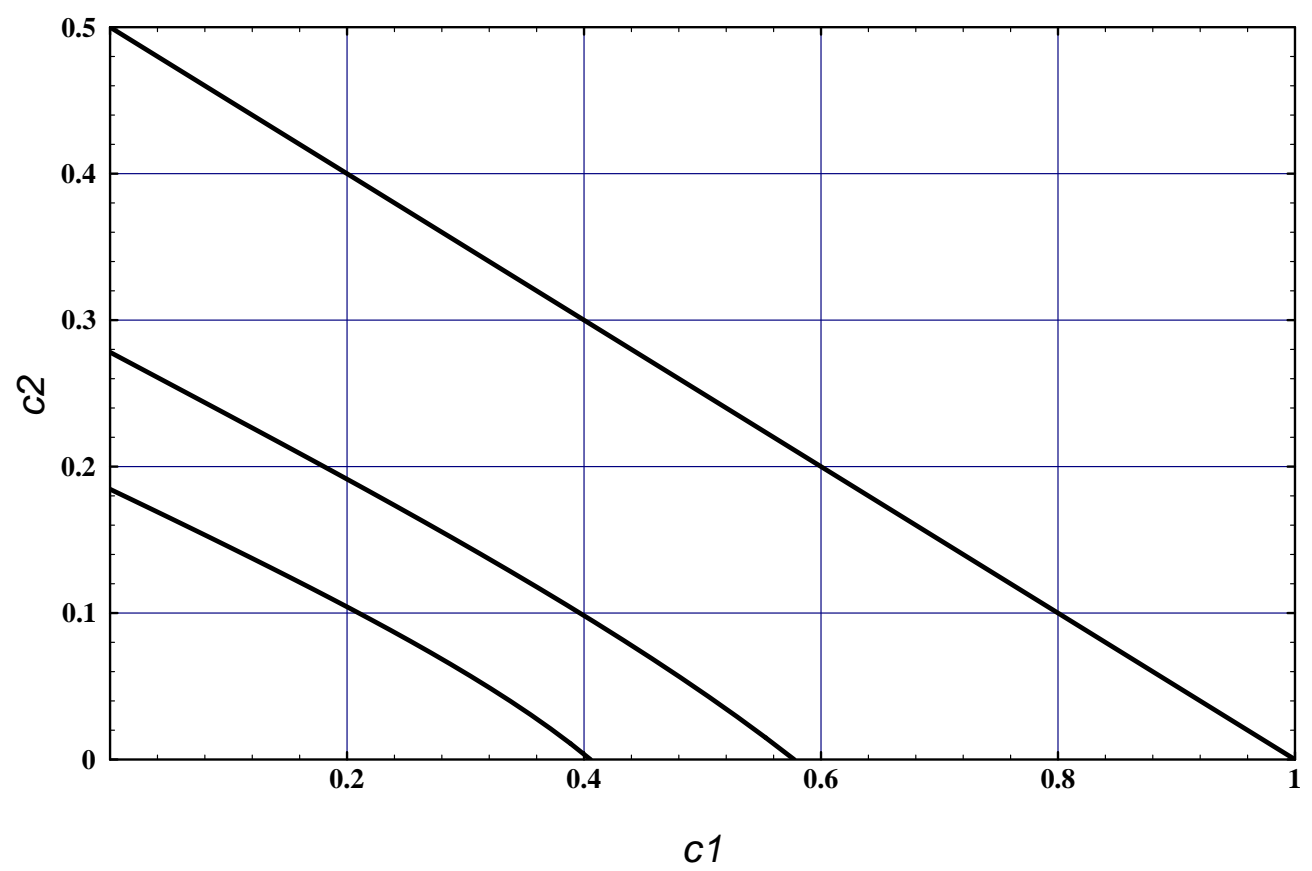

Figure 4: Moment conditions for $\mathrm{HARCH}(2)$ processes. The straight line on the right represents the boundary for the 2nd moment condition. The middle curve is the boundary for the existence of a finite 4th moment. The left curve represents the boundary for the existence condition of the 6th moment.

In the case of interest, the 4 th moment, we get

$$
3 c_{1}^{2}<1 \Rightarrow c_{1}^{2}<\frac{1}{3}
$$

the same condition as in (5.9) if $c_{2}=0$ (and $a_{2}=3$ ).

The fact that (5.9) is also sufficient for the existence of the 4th moment will follow from the general theory for $\mathrm{HARCH}(2)$ models to which we now turn.

\section{6 (2m)th moment of HARCH(2)}

\subsection{The system of equations}

To get a necessary condition for the existence of a stationary distribution of the HARCH(2) process with a finite $(2 m)$ th moment, $m \geq 2$, we denote

$$
\begin{aligned}
& M_{0}=\mathrm{E}\left(r_{n}^{2 m}\right), \\
& M_{i}=\mathrm{E}\left(r_{n}^{2(m-i)} r_{n-1}^{2 i}\right), \quad i=1, \ldots, m-1
\end{aligned}
$$

under stationarity conditions, in which case there is no dependence on $n$. If the $(2 m)$ th moment is finite, then all the moments of smaller orders are finite, $a_{m}<\infty$, and $M_{0}, M_{1}, \ldots, M_{m-1}$ above are all 
finite. Observe that

$$
\mathrm{E}\left(r_{n}^{2 m}\right)=\mathrm{E}\left(\sigma_{n}^{2 m}\right) \mathrm{E}\left(\varepsilon_{n}^{2 m}\right)
$$

and so using (6.2) and $a_{m}=\mathrm{E}\left(\varepsilon_{n}^{2 m}\right)$ we have that

$$
\begin{aligned}
M_{0}= & \mathrm{E}\left(r_{n}^{2 m}\right)=a_{m} \mathrm{E}\left[\left(c_{0}+c_{1} r_{n-1}^{2}+c_{2}\left(r_{n-1}+r_{n-2}\right)^{2}\right)^{m}\right] \\
= & a_{m} \mathrm{E}\left[\left(c_{0}+\left(c_{1}+c_{2}\right) r_{n-1}^{2}+c_{2} r_{n-2}^{2}+2 c_{2} r_{n-1} r_{n-2}\right)^{m}\right] \\
= & a_{m} \sum_{j=1}^{m}\left(\begin{array}{c}
m \\
j
\end{array}\right) c_{0}^{j} \mathrm{E}\left[\left(\left(c_{1}+c_{2}\right) r_{n-1}^{2}+c_{2} r_{n-2}^{2}+2 c_{2} r_{n-1} r_{n-2}\right)^{m-j}\right]+ \\
& +a_{m} \mathrm{E}\left[\left(\left(c_{1}+c_{2}\right) r_{n-1}^{2}+c_{2} r_{n-2}^{2}+2 c_{2} r_{n-1} r_{n-2}\right)^{m}\right] \\
& \equiv a_{m} \vartheta_{0, m}+a_{m} \mathrm{E}\left[\left(\left(c_{1}+c_{2}\right) r_{n-1}^{2}+c_{2} r_{n-2}^{2}+2 c_{2} r_{n-1} r_{n-2}\right)^{m}\right],
\end{aligned}
$$

where $\vartheta_{0, m}$ involves only moments of order less than $2 \mathrm{~m}$. Moreover, we have:

$$
\begin{aligned}
\mathrm{E}[ & \left.\left(\left(c_{1}+c_{2}\right) r_{n-1}^{2}+c_{2} r_{n-2}^{2}+2 c_{2} r_{n-1} r_{n-2}\right)^{m}\right]= \\
& =\sum_{j=0}^{[m / 2]}\left(\begin{array}{c}
m \\
2 j
\end{array}\right)\left(2 c_{2}\right)^{2 j} \mathrm{E}\left[\left(r_{n-1}^{2 j} r_{n-2}^{2 j}\right)\left(\left(c_{1}+c_{2}\right) r_{n-1}^{2}+c_{2} r_{n-2}^{2}\right)^{m-2 j}\right] \\
& =\sum_{j=0}^{[m / 2]}\left(\begin{array}{l}
m \\
2 j
\end{array}\right)\left(2 c_{2}\right)^{2 j} \mathrm{E}\left[\left(r_{n-1}^{2 j} r_{n-2}^{2 j}\right) \sum_{i=0}^{m-2 j}\left(\begin{array}{c}
m-2 j \\
i
\end{array}\right)\left(c_{1}+c_{2}\right)^{i} c_{2}^{m-2 j-i} r_{n-1}^{2 i} r_{n-2}^{2(m-2 j-i)}\right] \\
& =\sum_{j=0}^{[m / 2]} \sum_{i=0}^{m-2 j} \frac{m !}{(2 j) ! i !(m-2 j-i) !} 2^{2 j}\left(c_{1}+c_{2}\right)^{i} c_{2}^{m-i} \mathrm{E}\left(r_{n-1}^{2(i+j)} r_{n-2}^{2(m-j-i)}\right) \\
& =\sum_{j=0}^{[m / 2]} \sum_{i=0}^{m-2 j} \frac{m !}{(2 j) ! i !(m-2 j-i) !} 2^{2 j}\left(c_{1}+c_{2}\right)^{i} c_{2}^{m-i} M_{m-(i+j)},
\end{aligned}
$$

where $M_{m} \equiv M_{0}$, and [.] is the entier function. By replacing (6.4) in (6.3), we obtain:

$$
M_{0}=a_{m} \vartheta_{0, m}+a_{m} \sum_{j=0}^{[m / 2]} \sum_{i=0}^{m-2 j} \frac{m !}{(2 j) ! i !(m-2 j-i) !} 2^{2 j}\left(c_{1}+c_{2}\right)^{i} c_{2}^{m-i} M_{m-(i+j)} .
$$

Furthermore, for every $i=1, \ldots, m-1$,

$$
M_{i}=\mathrm{E}\left(r_{n}^{2(m-i)} r_{n-1}^{2 i}\right)=\mathrm{E}\left(\varepsilon_{n}^{2(m-i)}\right) \mathrm{E}\left(r_{n-1}^{2 i} \sigma_{n}^{2(m-i)}\right)
$$




$$
\begin{aligned}
& =a_{m-i} \mathrm{E}\left[r_{n-1}^{2 i}\left(c_{0}+\left(c_{1}+c_{2}\right) r_{n-1}^{2}+c_{2} r_{n-2}^{2}+2 c_{2} r_{n-1} r_{n-2}\right)^{m-i}\right] \\
& =a_{m-i} \sum_{j=1}^{m-i}\left(\begin{array}{c}
m-i \\
j
\end{array}\right) c_{0}^{j} \mathrm{E}\left[r_{n-1}^{2 i}\left(\left(c_{1}+c_{2}\right) r_{n-1}^{2}+c_{2} r_{n-2}^{2}+2 c_{2} r_{n-1} r_{n-2}\right)^{m-i-j}\right] \\
& \quad+a_{m-i} \mathrm{E}\left[r_{n-1}^{2 i}\left(\left(c_{1}+c_{2}\right) r_{n-1}^{2}+c_{2} r_{n-2}^{2}+2 c_{2} r_{n-1} r_{n-2}\right)^{m-i}\right] \\
& \quad a_{m-i} \vartheta_{i, m}+a_{m-i} \mathrm{E}\left[r_{n-1}^{2 i}\left(\left(c_{1}+c_{2}\right) r_{n-1}^{2}+c_{2} r_{n-2}^{2}+2 c_{2} r_{n-1} r_{n-2}\right)^{m-i}\right],
\end{aligned}
$$

where $\vartheta_{i, m}$ involves only moments of orders less than $2 m$. Now,

$$
\begin{aligned}
\mathrm{E}\left[r_{n-1}^{2 i}\left(\left(c_{1}+c_{2}\right) r_{n-1}^{2}+c_{2} r_{n-2}^{2}+2 c_{2} r_{n-1} r_{n-2}\right)^{m-i}\right]= \\
=\sum_{j=0}^{\left[\frac{m-i}{2}\right]}\left(2 c_{2}\right)^{2 j}\left(\begin{array}{c}
m-i \\
2 j
\end{array}\right) \mathrm{E}\left[r_{n-1}^{2(i+j)} r_{n-2}^{2 j}\left(\left(c_{1}+c_{2}\right) r_{n-1}^{2}+c_{2} r_{n-2}^{2}\right)^{m-i-2 j}\right]= \\
=\sum_{j=0}^{\left[\frac{m-i}{2}\right]}\left(2 c_{2}\right)^{2 j}\left(\begin{array}{c}
m-i \\
2 j
\end{array}\right) \sum_{d=0}^{m-i-2 j}\left(\begin{array}{c}
m-i-2 j \\
d
\end{array}\right)\left(c_{1}+c_{2}\right)^{d} c_{2}^{m-i-2 j-d} . \\
=\sum_{j=0}^{\left[\frac{m-i}{2}\right]} \sum_{d=0}^{m-i-2 j} \frac{(m-i) !}{(2 j) ! d !(m-i-2 j-d) !} 2^{2 j}\left(c_{1}+c_{2}\right)^{d} c_{2}^{m-i-d} M_{m-(i+j+d)}
\end{aligned}
$$

Therefore,

$$
\begin{aligned}
& M_{i}=a_{m-i} \vartheta_{i, m}+a_{m-i} \sum_{j=0}^{\left[\frac{m-i}{2}\right]} \sum_{d=0}^{m-i-2 j} \frac{(m-i) !}{(2 j) ! d !(m-i-2 j-d) !} \cdot \\
& \cdot 2^{2 j}\left(c_{1}+c_{2}\right)^{d} c_{2}^{m-i-d} M_{m-(i+j+d)}, \quad i=1, \ldots, m-1 .
\end{aligned}
$$

Equations (6.5) and (6.8) form a system of $m$ equations with $m$ unknowns $\left(M_{0}, M_{1}, \ldots, M_{m-1}\right)$. Let us call this system $\varrho_{m}$.

\subsection{The main theorem: necessary and sufficient moment conditions for HARCH(2)}

The following theorem presents a general result on the existence of a stationary version of the $\mathrm{HARCH}(2)$ process with a finite $(2 m)$ th moment. 
Theorem 1 The HARCH(2) process has a stationary version with a finite ( $2 m)$ th moment, if and only if, for every $j=1, \ldots, m$, the system $\varrho_{j}$ has a positive solution, with $\vartheta_{i, j}, j=2, \ldots, m, i=0, \ldots, j-1$ determined by solving $\varrho_{1}, \ldots, \varrho_{j-1}, j=2, \ldots, m$. If this is the case, then $\varrho_{m}$ has a unique solution given by (6.1).

Proof The proof is by induction on $m$. For $m=1$, we have already proved this statement. Assume now that this statement is also true for $m \geq 1$, and let us prove it for $m+1$.

Assume first that the the process is stationary, and $(2(m+1))$ th moment is finite. Then the $(2 m)$ th moment is finite as well, and so by the assumption of the induction, $\varrho_{1}, \ldots, \varrho_{m}$ all have a unique (positive) solution given by (6.1), and we use these solutions to compute $\vartheta_{i, m+1}$ for $i=0,1, \ldots, m$. Since $M_{0}, M_{1}, \ldots, M_{m}$, defined in (6.1), obviously yield a positive solution to $\varrho_{m+1}$, we only need to prove that this solution is unique.

Suppose, first of all, that there is a nonnegative solution $\underline{y}$ to $\varrho_{m+1}$, such that $y_{i}<M_{i}$ for at least one $i=0, \ldots, m$. We now proceed as follows: take a nonstationary HARCH(2) with the same coefficients, beginning with $r_{0}=r_{1}=0$. Observe that all the moments of the type $\mathrm{E}\left(r_{n}^{2 j} r_{n-1}^{2 k}\right), 0 \leq j+k \leq m$, are for $n=1$ less or equal to their corresponding stationary values (this is just the positivity of the moments), and

$$
\mathrm{E}\left(r_{n}^{2(m+1-k)} r_{n-1}^{2 k}\right) \leq y_{k}, \quad \text { with } k=0,1, \ldots, m
$$

(once again this is just the nonnegativity of $\underline{y}$ ). Since $\varrho_{1}, \ldots, \varrho_{k+1}$ are systems of equations with nonnegative coefficients, we conclude that the above is true for all $n \geq 1$.

Since we have assumed that the $\mathrm{HARCH}(2)$ process $\left(r_{n}\right)$ has a stationary version, it follows immediately that our Markov chain $\left(r_{n-1}, r_{n}\right)$ has a stationary distribution. Moreover, we have proved that the existence of a finite 2nd moment implies (4.5), which yields that the Markov chain $\left(r_{n-1}, r_{n}\right)$ is positive Harris recurrent. Therefore by Theorem 13.0.1 of (Meyn and Tweedie, 1990) we conclude that $\left(r_{n-1}, r_{n}\right)$ converges weakly to its stationary version. Therefore, so do the products $r_{n}^{2(m+1-k)} r_{n-1}^{2 k}, k=0,1, \ldots, m$. Hence, by Fatou's lemma, each $M_{k}$ does not exceed the lowest subsequential limit of $E\left(r_{n}^{2(m+1-k)} r_{n-1}^{2 k}\right)$, and so $M_{k} \leq y_{k}, k=0,1, \ldots, m$, which contradicts our assumption on $\underline{y}$.

Therefore $\underline{m}$ given by (6.1), is the smallest nonnegative solution of $\varrho_{m+1}$ and, if $\varrho_{m+1}$ has another nonnegative solution $\underline{y}$, we must have $y_{i} \geq M_{i}$ for all $i=0,1, \ldots, m$, and $y_{i}>M_{i}$ for at least one $i$. Thus for any $\alpha>0, \underline{y}_{\alpha}=(1+\alpha) \underline{m}-\alpha \underline{y}$ is yet another solution to $\varrho_{m+1}$. However, if $\alpha$ is small enough, we have $\underline{y}_{\alpha} \geq 0$, and some components of $\underline{y}_{\alpha}$ will be less then those of $\underline{m}$. This contradicts the already established fact that $\underline{m}$ is the smallest nonnegative solution to $\varrho_{m+1}$. Therefore, $\underline{m}$ is the only nonnegative solution of $\varrho_{m+1}$.

Suppose now that $\varrho_{m+1}$ has another, not necessary nonnegative, solution $\underline{y}$. Consider $\underline{y}_{\alpha}$ above. For all $|\alpha|$ small enough, $\underline{y}_{\alpha} \geq 0$, and for no $\alpha \neq 0$ it is equal to $\underline{m}$. Therefore, $\underline{m}$ is the only solution of $\varrho_{m+1}$.

In the opposite direction, assume that the system $\varrho_{j}$ has a positive solution for all $j=1, \ldots, m+1$. By the assumption of the induction, we know that the model has a stationary distribution with finite $(2 m)$ th moment, and, as above, is positive Harris recurrent. Once again, set $r_{0}=r_{1}=0$, and observe that all the moments of the type $\mathrm{E}\left(r_{n}^{2 i} r_{n-1}^{2 k}\right), 0 \leq i+k \leq m+1$, do not exceed the corresponding solution of the systems $\varrho_{1}, \ldots, \varrho_{k+1}$ for $n=1$, and so, by the nonnegativity of the coefficients of these systems of equations, for each $n \geq 1$. By Fatou's lemma, we conclude that the stationary version of $\mathrm{E}\left(r_{n}^{2(m+1)}\right)$ does not exceed the corresponding solution of $\varrho_{m+1}$, and so is finite.

This completes the proof of Theorem 1. 


\subsection{An explicit necessary and an explicit sufficient condition}

We now move to establish some more explicit necessary conditions for the existence of the $(2 \mathrm{~m})$ th moment of the HARCH(2) models. First of all, it follows from (6.5) $(i=j=0, i=m, j=0)$ that:

$$
M_{0}>a_{m}\left(c_{2}^{m}+\left(c_{1}+c_{2}\right)^{m}\right) M_{0}
$$

implying that

$$
\left(c_{1}+c_{2}\right)^{m}+c_{2}^{m}<\frac{1}{a_{m}}
$$

is a necessary condition for the existence of a finite $(2 m)$ th moment, $m=1,2, \ldots$. We can get a stricter necessary condition. It follows from (6.8) with $j=0, d=m-i$ that:

$$
M_{i}>a_{m-i}\left(c_{1}+c_{2}\right)^{m-i} M_{0}, \quad i=1, \ldots, m-1 .
$$

We rewrite (6.5) in the form

$$
\begin{aligned}
M_{0}=a_{m} \vartheta_{0, m}+a_{m} & \sum_{d=0}^{m} M_{d} \cdot \\
& \cdot \sum_{j=0}^{\operatorname{Min}[d,(m-d)]} 2^{2 j}\left(c_{1}+c_{2}\right)^{m-d-j} c_{2}^{d+j} \frac{m !}{(2 j) !(m-d-j) !(d-j) !},
\end{aligned}
$$

and substituting (6.11) into (6.12) we obtain:

$$
\begin{aligned}
& M_{0}>a_{m} M_{0}\left[\left(c_{1}+c_{2}\right)^{m}+c_{2}^{m}\right]+ \\
& +a_{m} \sum_{d=1}^{m-1} M_{d} \sum_{j=0}^{\operatorname{Min}[d,(m-d)]} 2^{2 j}\left(c_{1}+c_{2}\right)^{m-d-j} c_{2}^{d+j} \frac{m !}{(2 j) !(m-d-j) !(d-j) !} \\
& >a_{m} M_{0}\left[\left(\left(c_{1}+c_{2}\right)^{m}+c_{2}^{m}\right)+\sum_{d=1}^{m-1} a_{m-d}\left(c_{1}+c_{2}\right)^{m-d} \cdot\right. \\
& \left.\cdot \sum_{j=0}^{\operatorname{Min}[d,(m-d)]} 2^{2 j}\left(c_{1}+c_{2}\right)^{m-d-j} c_{2}^{d+j} \frac{m !}{(2 j) !(m-d-j) !(d-j) !}\right] .
\end{aligned}
$$

A necessary condition for existence of a finite $(2 m)$ th moment then is

$$
\left(c_{1}+c_{2}\right)^{m}+c_{2}^{m}+\sum_{d=1}^{m-1} a_{m-d} \cdot \sum_{j=0}^{\operatorname{Min}[d,(m-d)]} 2^{2 j}\left(c_{1}+c_{2}\right)^{2(m-d)-j} c_{2}^{d+j} \frac{m !}{(2 j) !(m-d-j) !(d-j) !}
$$




$$
<\frac{1}{a_{m}}, \quad m=1,2, \ldots
$$

Now we switch to computing a more explicit sufficient condition for the existence of a $(2 m)$ th moment of the HARCH(2) models. We claim that

$$
\left(c_{1}+4 c_{2}\right)^{m}<\frac{1}{a_{m}}
$$

is such a sufficient condition. Indeed, define

$$
V(x, y)=\alpha x^{2 m}+y^{2 m}, \quad 0<\alpha<1 .
$$

It is enough to prove that, under (6.15), with a suitable choice of $\alpha$ we have

$$
V(x, y)-P V(x, y) \geq \theta\left(x^{2 m}+y^{2 m}\right), \text { with } \theta>0
$$

outside of a compact set, as in subsection 4.2. Observe that

$$
\begin{gathered}
V(x, y)-P V(x, y)=\left(\alpha x^{2 m}+y^{2 m}\right)-\left(\alpha y^{2 m}+a_{m}\left(c_{0}+c_{1} y^{2}+c_{2}(x+y)^{2}\right)^{m}\right)= \\
=\alpha x^{2 m}+(1-\alpha) y^{2 m}-a_{m}\left(c_{1} y^{2}+c_{2}(x+y)^{2}\right)^{m}-\gamma(x, y)
\end{gathered}
$$

where $\gamma(x, y)$ is a polynomial of a lower order. It is, therefore, enough to prove that there is an $\alpha$ such that for all $x, y$ not both equal to zero,

$$
\alpha x^{2 m}+(1-\alpha) y^{2 m}-a_{m}\left(c_{1} y^{2}+c_{2}(x+y)^{2}\right)^{m}>\theta\left(x^{2 m}+y^{2 m}\right), \quad \text { with } \theta>0 \text {. }
$$

By the homogeneity of the terms in the inequality (6.19), it suffices to show that for all $(x, y) \neq(0,0)$,

$$
\alpha x^{2 m}+(1-\alpha) y^{2 m}>a_{m}\left(c_{1} y^{2}+c_{2}(x+y)^{2}\right)^{m} .
$$

We are finally ready to specify $\alpha$. Let

$$
\alpha=\frac{1-a_{m}^{1 / m} c_{1}}{2}
$$

We have, by convexity of the function $f(t)=t^{m}$,

$$
\alpha x^{2 m}+(1-\alpha) y^{2 m} \geq\left(\alpha x^{2}+(1-\alpha) y^{2}\right)^{m},
$$

and so inequality (6.20) will follow once we prove that

$$
\alpha x^{2}+(1-\alpha) y^{2}>a_{m}^{1 / m}\left(c_{1} y^{2}+c_{2}(x+y)^{2}\right) .
$$

We have

$$
\alpha x^{2}+(1-\alpha) y^{2}-a_{m}^{1 / m}\left(c_{1} y^{2}+c_{2}(x+y)^{2}\right)=
$$




$$
=x^{2}\left(\alpha-a_{m}^{1 / m} c_{2}\right)-2 a_{m}^{1 / m} c_{2} x y+\left(1-\alpha-\left(c_{1}+c_{2}\right) a_{m}^{1 / m}\right) y^{2} .
$$

Using (6.15) and (6.21), we can write

$$
\alpha-a_{m}^{1 / m} c_{2}=\frac{1-a_{m}^{1 / m}\left(c_{1}+2 c_{2}\right)}{2}>0 .
$$

Moreover,

$$
\begin{gathered}
(1-\alpha)-\left(c_{1}+c_{2}\right) a_{m}^{1 / m}=\frac{1+a_{m}^{1 / m} c_{1}}{2}-a_{m}^{1 / m}\left(c_{1}+c_{2}\right)= \\
=\frac{1-a_{m}^{1 / m}\left(c_{1}+2 c_{2}\right)}{2}=\alpha-a_{m}^{1 / m} c_{2}>0 .
\end{gathered}
$$

Finally, by (6.25) and (6.26),

$$
\begin{aligned}
\left(\alpha-a_{m}^{1 / m} c_{2}\right)((1-\alpha) & \left.-\left(c_{1}+c_{2}\right) a_{m}^{1 / m}\right)-a_{m}^{2 / m} c_{2}^{2}= \\
= & \left(\frac{1-a_{m}^{1 / m}\left(c_{1}+2 c_{2}\right)}{2}\right)^{2}-a_{m}^{2 / m} c_{2}^{2}>0
\end{aligned}
$$

because, by (6.15),

$$
\frac{1-a_{m}^{1 / m}\left(c_{1}+4 c_{2}\right)}{2}>0
$$

This proves the inequality (6.23), and so the condition (6.15) is a sufficient condition for the existence of a $(2 m)$ th moment.

\section{Stationarity condition with finite 2 nd moment for $\mathrm{HARCH}(\mathrm{k})$}

We now discuss the more difficult case: conditions for stationarity and finite moments of the general $\mathrm{HARCH}(\mathrm{k})$ process, and we start with the easiest and most explicit part: finiteness of the second moment. The following condition has been shown in (Müller et al., 1995) (relation (3.6)) to be necessary for existence of a finite second moment:

$$
\sum_{j=1}^{k} j c_{j}<\frac{1}{a_{1}}
$$

We will prove now that this condition is also sufficient.

Let $\alpha_{1}=1$. A simple inductive argument establishes that we can choose $\alpha_{2}, \ldots, \alpha_{k}$ in such a way that

$$
a_{1}\left(S_{i}+\ldots+S_{k}\right)<\alpha_{i}<\alpha_{i-1}-a_{1} S_{i-1}, \quad i=2, \ldots, k
$$


where $S_{i}=c_{i}+\ldots+c_{k}$ with $i=1, \ldots, k$.

Indeed, a condition equivalent to (7.1) is

$$
\sum_{j=1}^{k} S_{j}<\frac{1}{a_{1}}
$$

and so $a_{1}\left(S_{2}+\ldots+S_{k}\right)<1-a_{1} S_{1}$, implying that there is a number strictly between the two, which we declare to be $\alpha_{2}$. Assuming that we have chosen $\alpha_{1}, \ldots, \alpha_{i}, i<k$, we have by (7.2),

$$
a_{1}\left(S_{i+1}+\ldots+S_{k}\right)<\alpha_{i}-a_{1} S_{i}
$$

allowing us to choose $\alpha_{i+1}$ in the interior of the above interval. Having chosen $\alpha_{1}, \ldots, \alpha_{k}$ as above, we define a function $V: \mathbb{R}^{k} \longrightarrow \mathbb{R}$ by

$$
V\left(x_{1}, \ldots, x_{k}\right)=\sum_{i=1}^{k} \alpha_{i} x_{i}^{2}+2 a_{1} \sum_{i=1}^{k} \sum_{j=i+1}^{k} \beta_{j} x_{i} x_{j}
$$

where

$$
\beta_{j}=S_{j}+\ldots+S_{k}, j=2, \ldots, k \text {. }
$$

We claim that $V\left(x_{1}, \ldots, x_{k}\right) \geq 0$. To this end we need to show that the matrix

$$
A=\left(\begin{array}{ccccc}
\alpha_{1} & a_{1} \beta_{2} & a_{1} \beta_{3} & \ldots . . & a_{1} \beta_{k} \\
a_{1} \beta_{2} & \alpha_{2} & a_{1} \beta_{3} & \ldots . . & a_{1} \beta_{k} \\
\cdot & & & & \\
\cdot & & & & \\
\cdot & & & & \\
a_{1} \beta_{k} & a_{1} \beta_{k} & a_{1} \beta_{k} & \ldots . . & \alpha_{k}
\end{array}\right)
$$

is non-negatively definite. For this, it suffices to note that $A$ is the covariance matrix of the following Gaussian random vector

$$
X_{i}=a_{1}^{1 / 2} \sum_{j=i}^{k} S_{j}^{1 / 2} G_{j}+\left(\alpha_{i}-a_{1}\left(S_{i}+\ldots+S_{k}\right)\right)^{1 / 2} U_{i}, \quad i=1, \ldots, k
$$

where $G_{1}, \ldots, G_{k}, U_{1}, \ldots, U_{k}$ are iid $N(0,1)$ random variables.

Now, we turn to the quantity needed for the different levels described in section 4.2.2:

$$
\begin{aligned}
& V\left(r_{n-k+1}, \ldots, r_{n}\right)- P V\left(r_{n-k+1}, \ldots, r_{n}\right)= \\
& \sum_{i=1}^{k} \alpha_{i} r_{n+1-i}^{2}+2 a_{1} \sum_{i=1}^{k} \sum_{j=i+1}^{k} \beta_{j} r_{n+1-i} r_{n+1-j}-
\end{aligned}
$$




$$
\begin{aligned}
& -E\left[\sum_{i=2}^{k} \alpha_{i} r_{n+2-i}^{2}+\alpha_{1} \varepsilon_{n+1}^{2}\left(c_{0}+\sum_{i=1}^{k} c_{i}\left(\sum_{j=1}^{i} r_{n+1-j}\right)^{2}\right)+\right. \\
& +2 a_{1} \sum_{i=2}^{k} \sum_{j=i+1}^{k} \beta_{j} r_{n+2-i} r_{n+2-j}+ \\
& \left.+2 \varepsilon_{n+1}\left(c_{0}+\sum_{i=1}^{k} c_{i}\left(\sum_{m=1}^{i} r_{n+1-m}\right)^{2}\right)^{1 / 2} \sum_{j=2}^{k} \beta_{j} r_{n+2-j}\right] .
\end{aligned}
$$

Using similar arguments as in the case $k=2$, we can simplify the above as

$$
\begin{aligned}
V\left(r_{n-k+1}, \ldots, r_{n}\right) & -P V\left(r_{n-k+1}, \ldots, r_{n}\right)= \\
& \sum_{i=1}^{k} \alpha_{i} r_{n+1-i}^{2}+2 a_{1} \sum_{i=1}^{k} \sum_{j=i+1}^{k} \beta_{j} r_{n+1-i} r_{n+1-j}- \\
& -\sum_{i=2}^{k} \alpha_{i} r_{n+2-i}^{2}-a_{1}\left(c_{0}-\sum_{i=1}^{k} c_{i}\left(\sum_{j=1}^{i} r_{n+1-j}\right)^{2}\right)- \\
& -2 a_{1} \sum_{i=2}^{k} \sum_{j=i+1}^{k} \beta_{j} r_{n+2-i} r_{n+2-j} .
\end{aligned}
$$

Setting $\alpha_{k+1}=\beta_{k+1}=0$, we can rewrite the expression in terms of the $S_{i}$ and we get

$$
\begin{aligned}
& V\left(r_{n-k+1}, \ldots, r_{n}\right)-P V\left(r_{n-k+1}, \ldots, r_{n}\right)= \\
& \sum_{i=1}^{k}\left(\alpha_{i}-\alpha_{i+1}-a_{1} S_{i}\right) r_{n+1-i}^{2}-2 a_{1} \sum_{i=1}^{k} \sum_{j=i+1}^{k}\left(\beta_{j}-\beta_{j+1}-S_{j}\right) r_{n+1-i} r_{n+1-j}-c_{0} .(7.1
\end{aligned}
$$

By (7.6), we conclude that

$$
V\left(r_{n-k+1}, \ldots, r_{n}\right)-P V\left(r_{n-k+1}, \ldots, r_{n}\right)=\sum_{i=1}^{k}\left(\alpha_{i}-\alpha_{i+1}-a_{1} S_{i}\right) r_{n+1-i}^{2}-c_{0}
$$

Let

$$
\vartheta_{i}=\alpha_{i}-\alpha_{i+1}-a_{1} S_{i}, \quad i=1, \ldots, k \text {. }
$$

It follows from (7.2) that $\vartheta_{i}>0, i=1, \ldots, k$, and so

$$
\Theta=\min _{i=1, . ., k} \vartheta_{i}>0
$$


Letting

$$
f\left(x_{1}, \ldots, x_{k}\right)=x_{1}^{2}+\ldots+x_{k}^{2}
$$

and denoting by $B_{a}(a>0)$ the sphere centered at 0 with radius $a$, we conclude from (7.12) that

$$
V\left(r_{n-k+1}, \ldots, r_{n}\right)-P V\left(r_{n-k+1}, \ldots, r_{n}\right) \geq \Theta f\left(x_{1}, \ldots, x_{k}\right) / 2-c_{0} 1_{B\left(2 c_{0} / \Theta\right)}\left(x_{1}, \ldots, x_{k}\right)
$$

for all $\left(x_{1}, \ldots, x_{k}\right)$. Therefore, all levels of Section 4.2 are satisfied for this Markov chain. That is, the stationary $\mathrm{HARCH}(\mathrm{k})$ process has, under the condition (7.1), finite second moments.

\section{Existence of the 4th moment of a general HARCH(k)}

\subsection{The system of equations}

Assume first, that the 2nd moments are finite. That is, we assume that the condition (7.1) holds and denote,

$$
\begin{aligned}
& L=\mathrm{E}\left(r_{n}^{2}\right), \\
& M_{0}=\mathrm{E}\left(r_{n}^{4}\right), \\
& M_{i}=\mathrm{E}\left(r_{n}^{2} r_{n-i}^{2}\right), \quad i=1, \ldots, k-1, \\
& n_{i j}=\mathrm{E}\left(r_{n}^{2} r_{n-i} r_{n-j}\right), \quad i=1, \ldots, k-2, \quad j=i+1, \ldots, k-1 .
\end{aligned}
$$

We have:

$$
n_{i j}=\mathrm{E}\left(r_{n}^{2} r_{n-i} r_{n-j}\right)=\mathrm{E}\left(\varepsilon_{n}^{2}\right) \mathrm{E}\left(\sigma_{n}^{2} r_{n-i} r_{n-j}\right)
$$

Recalling that $\mathrm{E}\left(\varepsilon_{n}^{2}\right)=a_{1}$, we can write the expression as:

$$
\begin{aligned}
n_{i j} & =a_{1} \mathrm{E}\left(\sigma_{n}^{2} r_{n-i} r_{n-j}\right)=a_{1} \mathrm{E}\left[r_{n-i} r_{n-j}\left(c_{0}+\sum_{l=1}^{k} c_{l}\left(\sum_{d=1}^{l} r_{n-d}\right)^{2}\right]\right. \\
& =a_{1} \sum_{l=1}^{k} c_{l} \mathrm{E}\left[r_{n-i} r_{n-j}\left(\sum_{d=1}^{l} r_{n-d}\right)^{2}\right] .
\end{aligned}
$$

We evaluate the expectation under the sum in (8.1) by examining different cases for the index $l$.

Case 1. $1 \leq l<i$.

$$
\mathrm{E}\left[r_{n-i} r_{n-j}\left(\sum_{d=1}^{l} r_{n-d}\right)^{2}\right]=\mathrm{E}\left[r_{n-i} r_{n-j} \sum_{d=1}^{l} r_{n-d}^{2}\right]+2 \sum_{d_{1}=1}^{l} \sum_{d_{2}=d_{1}+1}^{l} \mathrm{E}\left[r_{n-i} r_{n-j} r_{n-d_{1}} r_{n-d_{2}}\right]
$$




$$
=\sum_{d=1}^{l} \mathrm{E}\left[r_{n-i} r_{n-j} r_{n-d}^{2}\right]=\sum_{d=1}^{l} n_{i-d, j-d},
$$

since the expectation of a product of only odd powers of $r_{n}$ is zero.

Case 2. $i \leq l<j$.

Here we have

$$
\begin{aligned}
\mathrm{E}\left[r_{n-i} r_{n-j}\left(\sum_{d=1}^{l} r_{n-d}\right)^{2}\right] & =\sum_{d=1}^{i-1} \mathrm{E}\left[r_{n-d}^{2} r_{n-i} r_{n-j}\right]+2 \sum_{d_{2}=i+1}^{l} \mathrm{E}\left[r_{n-i}^{2} r_{n-j} r_{n-d_{2}}\right] \\
& =\sum_{d=1}^{i-1} n_{i-d, j-d}+2 \sum_{d=i+1}^{l} n_{d-i, j-i} .
\end{aligned}
$$

Case 3. $j \leq l \leq k$.

Similarly to the above we have

$$
\begin{gathered}
\mathrm{E}\left[r_{n-i} r_{n-j}\left(\sum_{d=1}^{l} r_{n-d}\right)^{2}\right]=\sum_{d=1}^{i-1} \mathrm{E}\left[r_{n-d}^{2} r_{n-i} r_{n-j}\right]+2 \sum_{d=i+1}^{l} \mathrm{E}\left[r_{n-i}^{2} r_{n-j} r_{n-d}\right]= \\
=\sum_{d=1}^{i-1} n_{i-d, j-d}+2 \sum_{d=i+1}^{j-1} n_{d-i, j-i}+2 M_{j-i}+2 \sum_{d=j+1}^{l} n_{j-i, d-i} .
\end{gathered}
$$

Putting together all three cases and using (8.1), we obtain an equation for the $n_{i j}$ 's:

$$
\begin{aligned}
\frac{1}{a_{1}} n_{i j} & =\sum_{l=1}^{i-1} c_{l} \sum_{d=1}^{l} n_{i-d, j-d}+\sum_{l=i}^{j-1} c_{l}\left(\sum_{d=1}^{i-1} n_{i-d, j-d}+2 \sum_{d=i+1}^{l} n_{d-i, j-i}\right)+ \\
& +\sum_{l=j}^{k} c_{l}\left(\sum_{d=1}^{i-1} n_{i-d, j-d}+2 \sum_{d=i+1}^{j-1} n_{d-i, j-i}+2 M_{j-i}+2 \sum_{d=j+1}^{l} n_{j-i, d-i}\right),
\end{aligned}
$$

with $1 \leq i<j \leq k-1$.

We now turn to finding an equation for $M_{0}$ :

$$
\begin{aligned}
M_{0} & =\mathrm{E}\left(r_{n}^{4}\right)=a_{2} \mathrm{E}\left(\sigma_{n}^{4}\right)=a_{2} \mathrm{E}\left[\left(c_{0}+\sum_{l=1}^{k} c_{l}\left(\sum_{d=1}^{l} r_{n-d}\right)^{2}\right)^{2}\right] \\
& =a_{2}\left[c_{0}^{2}+2 c_{0} \sum_{l=1}^{k} c_{l} \mathrm{E}\left[\left(\sum_{d=1}^{l} r_{n-d}\right)^{2}\right]+\mathrm{E}\left[\left(\sum_{l=1}^{k} c_{l}\left(\sum_{d=1}^{l} r_{n-d}\right)^{2}\right)^{2}\right]\right]
\end{aligned}
$$




$$
=a_{2}\left[c_{0}^{2}+2 c_{0} \sum_{l=1}^{k} c_{l} l L+\mathrm{E}\left[\left(\sum_{l=1}^{k} c_{l} \sum_{d=1}^{l} r_{n-d}^{2}+2 \sum_{l=1}^{k} c_{l} \sum_{d_{1}=1}^{l} \sum_{d_{2}=d_{1}+1}^{l} r_{n-d_{1}} r_{n-d_{2}}\right)^{2}\right]\right] .
$$

We continue to develop the right hand side of the equation by introducing more partial sums,

$$
\begin{aligned}
\frac{M_{0}}{a_{2}}= & c_{0}^{2}+2 c_{0} L \sum_{l=1}^{k} l c_{l}+\mathrm{E}\left[\left(\sum_{l=1}^{k} c_{l} \sum_{d=1}^{l} r_{n-d}^{2}\right)^{2}\right]+ \\
& +4 \sum_{l_{1}=1}^{k} \sum_{l_{2}=1}^{k} c_{l_{1}} c_{l_{2}} \sum_{d_{3}=1}^{l_{1}} \sum_{d_{1}=1}^{l_{2}} \sum_{d_{2}=d_{1}+1}^{l_{2}} \mathrm{E}\left(r_{n-d_{3}}^{2} r_{n-d_{1}} r_{n-d_{2}}\right)+ \\
& +4 \sum_{l_{1}=1}^{k} \sum_{l_{2}=1}^{k} c_{l_{1}} c_{l_{2}} \sum_{d_{1}=1}^{l_{1}} \sum_{d_{2}=d_{1}+1}^{l_{1}} \sum_{d_{3}=1}^{l_{2}} \sum_{d_{4}=d_{3}+1}^{l_{2}} \mathrm{E}\left(r_{n-d_{1}} r_{n-d_{2}} r_{n-d_{3}} r_{n-d_{4}}\right) \\
= & c_{0}^{2}+2 c_{0} L \sum_{l=1}^{k} l c_{l}+\mathrm{E}\left[\left(\sum_{d=1}^{k} r_{n-d}^{2} \sum_{l=d}^{k} c_{l}\right)^{2}\right]+ \\
& +4 \sum_{l_{1}=1}^{k} \sum_{l_{2}=1}^{k} c_{l_{1}} c_{l_{2}} \sum_{d_{1}=1}^{l_{1}} \sum_{d_{2}=d_{1}+1}^{l_{2}} \sum_{d_{3}=d_{2}+1}^{l_{2}} n_{d_{2}-d_{1}, d_{3}-d_{1}}+ \\
& +4 \sum_{l_{1}=1}^{k} \sum_{l_{2}=1}^{k} c_{l_{1}} c_{l_{2}} \sum_{d_{1}=1}^{\operatorname{Min}_{1}\left[l_{1}, l_{2}\right]} \sum_{d_{2}=d_{1}+1}^{l_{1}} \sum_{d_{3}=d_{1}+1}^{l_{2}} \mathrm{E}\left(r_{n-d_{1}}^{2} r_{n-d_{2}} r_{n-d_{3}}\right) .
\end{aligned}
$$

Using now the definitions we set at the beginning of this section, we get

$$
\begin{aligned}
\frac{M_{0}}{a_{2}}= & c_{0}^{2}+2 c_{0} L \sum_{l=1}^{k} l c_{l}+M_{0} \sum_{d=1}^{k}\left(\sum_{l=d}^{k} c_{l}\right)^{2}+ \\
& +2 \sum_{l_{1}=1}^{k} \sum_{l_{2}=1}^{k} c_{l_{1}} c_{l_{2}} \sum_{d_{1}=1}^{l_{1}} \sum_{d_{2}=d_{1}+1}^{l_{2}} M_{d_{2}-d_{1}}+ \\
& +4 \sum_{l_{1}=1}^{k} \sum_{l_{2}=1}^{k} c_{l_{1}} c_{l_{2}} \sum_{d_{1}=1}^{l_{1}} \sum_{d_{2}=d_{1}+1}^{l_{2}} \sum_{d_{3}=d_{2}+1}^{l_{2}} n_{d_{2}-d_{1}, d_{3}-d_{1}}+ \\
& +4 \sum_{l_{1}=1}^{k} \sum_{l_{2}=1}^{k} c_{l_{1}} c_{l_{2}} \sum_{d_{1}=1}^{\operatorname{Min}\left[l_{1}, l_{2}\right]} \sum_{d_{2}=d_{1}+1}^{l_{1}} \sum_{d_{3}=d_{2}+1}^{l_{2}} n_{d_{2}-d_{1}, d_{3}-d_{1}}+ \\
& +4 \sum_{l_{1}=1}^{k} \sum_{l_{2}=1}^{k} c_{l_{1}} c_{l_{2}} \sum_{d_{1}=1}^{\operatorname{Min}\left[l_{1}, l_{2}\right]} \sum_{d_{2}=d_{1}+1}^{l_{2}} \sum_{d_{3}=d_{2}+1}^{l_{1}} n_{d_{2}-d_{1}, d_{3}-d_{1}}
\end{aligned}
$$


This is an equation for the variable $M_{0}$.

Finally, we study the equations for the variables $M_{i}{ }^{\prime}$ s with the index $i=1, \ldots, k-1$ :

$$
\begin{aligned}
M_{i}= & \mathrm{E}\left(r_{n}^{2} r_{n-i}^{2}\right)=\mathrm{E}\left(\sigma_{n}^{2} r_{n-i}^{2}\right) \mathrm{E}\left(\varepsilon_{n}^{2}\right)=a_{1} \mathrm{E}\left(\sigma_{n}^{2} r_{n-i}^{2}\right) \\
= & a_{1} \mathrm{E}\left[r_{n-i}^{2}\left(c_{0}+\sum_{l=1}^{k} c_{l}\left(\sum_{d=1}^{l} r_{n-d}\right)^{2}\right)\right]=a_{1} c_{0} L+a_{1} \sum_{l=1}^{k} c_{l} \mathrm{E}\left[r_{n-i}^{2}\left(\sum_{d=1}^{l} r_{n-d}\right)^{2}\right] \\
= & a_{1} c_{0} L+a_{1} \sum_{l=1}^{i-1} c_{l} \mathrm{E}\left[r_{n-i}^{2} \sum_{d=1}^{l} r_{n-d}^{2}\right]+a_{1} \sum_{l=i}^{k} c_{l} \mathrm{E}\left[r_{n-i}^{2} \sum_{d=1}^{l} r_{n-d}^{2}\right]+ \\
& +2 a_{1} \sum_{l=i}^{k} c_{l} \mathrm{E}\left[r_{n-i}^{2} \sum_{d_{1}=1}^{l} r_{n-d_{1}} \sum_{d_{2}=d_{1}+1}^{l} r_{n-d_{2}}\right] .
\end{aligned}
$$

Using again the definitions at the beginning of the section, we obtain the equations for $M_{i}$ :

$$
\begin{aligned}
\frac{M_{i}}{a_{1}}= & c_{0} L+\sum_{l=1}^{i-1} c_{l} \sum_{d=1}^{l} M_{i-d}+\sum_{l=i}^{k} c_{l}\left(M_{0}+\sum_{d=1}^{i-1} M_{i-d}+\sum_{d=i+1}^{l} M_{d-i}\right)+ \\
& +2 \sum_{l=i}^{k} c_{l} \sum_{d_{1}=i+1}^{l} \sum_{d_{2}=d_{1}+1}^{l} n_{d_{1}-i, d_{2}-i} .
\end{aligned}
$$

Overall, equations (8.5), (8.6) and (8.7) give a system of $\frac{k^{2}-k+2}{2}$ equations with as many unknowns. We also mention that it follows from Theorem 2 below that existence of a positive solution to this system of equations, constitutes, together with (4.5), a necessary and sufficient condition for the existence of a stationary version of the $\mathrm{HARCH}(\mathrm{k})$ process with a finite 4 th moment. The following two subsections give more explicit conditions.

\subsection{An explicit necessary condition for the existence of 4th moments of HARCH(k)}

We start with showing that, if the 4th moment is finite, then we must have

$$
n_{i j} \geq 0, \quad \text { with } i=1, \ldots, k-2 \text { and } j=i+1, . ., k-1 .
$$

To this end, observe that

$$
P\left(\tau_{n} \geq 0, \tau_{n-1} \geq 0, \ldots, \tau_{n-k+1} \geq 0\right) \geq 2^{-k}>0
$$

for every $n \geq k$.

Define a function

$$
f\left(x_{1}, \ldots, x_{k}\right)=x_{1}^{4}+x_{2}^{4}+\ldots+x_{k}^{4} .
$$


If the 4th moment of $\mathrm{HARCH}(\mathrm{k})$ is finite, we conclude from Theorem 14.3.3 of (Meyn and Tweedie, 1990) that the set $\mathcal{S}_{f}$ of f-regular points has probability 1 (under the steady state), and so by (8.9), it follows that there is an f-regular point $\left(x_{1}, \ldots, x_{k}\right)$ with $x_{j} \geq 0, j=1, \ldots, k$. We then set

$$
r_{n}=x_{n}, \quad n=1, \ldots, k,
$$

and we observe that

$$
n_{i j}^{(n)}=\mathrm{E}\left(r_{n}^{2} r_{n-i} r_{n-j}\right) \geq 0, \quad i=1, \cdots k-2, j=i+1, \cdots, k-1
$$

for $n=k$. We claim that (8.12) holds for all $n \geq k$. The proof is by induction on $n$. We have seen that (8.12) holds for $n=k$. Assume that it holds for all $k \leq l \leq n$ and let us prove it for $n+1$. As in equation 8.5 , we have,

$$
\begin{aligned}
& \frac{n_{i j}^{(n+1)}}{a_{1}}=\sum_{l=1}^{i-1} c_{l} \sum_{d=1}^{l} n_{i-d, j-d}^{\left(k_{1}(i, j, d)\right)}+\sum_{l=i}^{j-1} c_{l}\left(\sum_{d=1}^{i-1} n_{i-d, j-d}^{\left(k_{2}(i, j, d)\right)}+2 \sum_{d=i+1}^{l} n_{d-i, j-i}^{\left(k_{3}(i, j, d)\right)}\right)+ \\
& +\sum_{l=j}^{k} c_{l}\left(\sum_{d=1}^{j-1} n_{i-d, j-d}^{\left(k_{4}(i, j, d)\right)}+2 \sum_{d=i+1}^{j-1} n_{d-i, j-i}^{\left(k_{5}(i, j, d)\right)}+2 \mathrm{E}\left(r_{n+1-i}^{2} r_{n+1-j}^{2}\right)+2 \sum_{d=j+1}^{l} n_{i-d, j-d}^{\left(k_{5}(i, j, d)\right)}\right),
\end{aligned}
$$

where

$$
k_{p}(i, j, d) \leq n, \quad p=1, \ldots, 5
$$

Therefore, by the assumption of the induction, $n_{i j}^{(n+1)} \geq 0$, and (8.12) has been proved. Let

$$
g\left(x_{1}, \ldots, x_{k}\right)=x_{k}^{2} x_{k-i} x_{k-j}, \quad i=1, \ldots, k-2, j=i+1, \ldots, k+1 .
$$

Observe that

$$
\left|g\left(x_{1}, \ldots, x_{k}\right)\right| \leq \max _{j \leq k}\left|x_{j}\right|^{4} \leq f\left(x_{1}, \ldots, x_{k}\right)
$$

Therefore, by Theorem 14.3.3 of (Meyn and Tweedie, 1990), $\mathrm{E}\left(r_{n}^{2} r_{n-i} r_{n-j}\right)$ converges to its stationary version, and so it follows by (8.12) that this stationary expectation is nonnegative. This proves (8.8).

The discussion above allows us to get explicit necessary conditions for the existence of the 4th moment of the HARCH(k) models. First of all, it follows from (8.6) that

$$
M_{0} \geq a_{2}\left[c_{0}^{2}+2 c_{0} M \sum_{l=1}^{k} l c_{l}+M_{0} \sum_{d=1}^{k}\left(\sum_{l=d}^{k} c_{l}\right)^{2}+2 \sum_{l_{1}=1}^{k} \sum_{l_{2}=1}^{k} c_{l_{1}} c_{l_{2}} \sum_{d_{1}=1}^{l_{1}} \sum_{d_{2}=d_{1}+1}^{l_{2}} M_{d_{2}-d_{1}}\right]
$$

and so

$$
M_{0} \geq a_{2}\left[c_{0}^{2}+2 c_{0} M \sum_{l=1}^{k} l c_{l}+M_{0} \sum_{d=1}^{k}\left(\sum_{l=d}^{k} c_{l}\right)^{2}+a_{1} \sum_{l_{1}=1}^{k} \sum_{l_{2}=1}^{k} c_{l_{1}} c_{l_{2}} \sum_{j=1}^{l_{2}-1} \operatorname{Min}\left[l_{1},\left(l_{2}-j\right)\right] M_{j}\right]
$$




$$
\geq a_{2}\left[c_{0}^{2}+2 c_{0} M \sum_{l=1}^{k} l c_{l}+M_{0} \sum_{d=1}^{k}\left(\sum_{l=d}^{k} c_{l}\right)^{2}+2 \sum_{j=1}^{k-1} M_{j} \sum_{l=1}^{k-j} l(2 k-2 l+1-j)\right] .
$$

Moreover, it follows from (8.7) that

$$
\begin{aligned}
\frac{M_{i}}{a_{1}} \geq c_{0} M & +\sum_{l=1}^{i-1} c_{l} \sum_{d=1}^{l} M_{i-d}+ \\
& +\sum_{l=i}^{k} c_{l}\left(\sum_{d=1}^{i-1} M_{i-d}+M_{0}+\sum_{d=i+1}^{l} M_{d-i}\right), \quad i=1, \ldots, k-1 .
\end{aligned}
$$

We immediately see from (8.16) that

$$
M_{0} \geq a_{2}\left[c_{0}^{2}+2 c_{0} M \sum_{l=1}^{k} l c_{l}+M_{0} \sum_{d=1}^{k}\left(\sum_{l=d}^{k} c_{l}\right)^{2}\right]
$$

and so

$$
\sum_{d=1}^{k}\left(\sum_{l=d}^{k} c_{l}\right)^{2}<\frac{1}{a_{2}}
$$

is a necessary condition for the existence of a finite 4th moment; but it is, clearly, insufficient. We can get a stricter necessary condition as follows. From (8.17), we know that for every $i=1, \ldots, k-1$,

$$
M_{i} \geq M_{0} a_{1} \sum_{l=i}^{k} c_{l}
$$

Substituting (8.19) into (8.16), we obtain

$$
M_{0} \geq a_{2}\left[c_{0}^{2}+2 c_{0} M \sum_{l=1}^{k} l c_{l}+M_{0} \sum_{d=1}^{k}\left(\sum_{l=d}^{k} c_{l}\right)^{2}+2 a_{1} M_{0} \sum_{j=1}^{k-1} \sum_{l=j}^{k} c_{j} \sum_{l=1}^{k-j} l(2 k-2 l+1-j)\right]
$$

and so a necessary condition for the existence of a finite 4 th moment is

$$
\sum_{d=1}^{k}\left(\sum_{l=d}^{k} c_{l}\right)^{2}+2 a_{1} \sum_{j=1}^{k-1} \sum_{l=j}^{k} c_{j} \sum_{l=1}^{k-j} l(2 k-2 l+1-j)<\frac{1}{a_{2}} .
$$

\subsection{A sufficient condition for the existence of the 4th moment of HARCH(k)}

We now move to derive some explicit sufficient conditions for the existence of a finite 4 th moment of a $\operatorname{HARCH}(\mathrm{k})$ model. Specifically, we claim that if

$$
\left(\sum_{j=1}^{k} j^{2} c_{j}\right)^{2}<\frac{1}{a_{2}}
$$


then a finite 4 th moment exists.

Indeed, if (8.22) holds, one can choose positive numbers $\alpha_{1}, \ldots, \alpha_{k-1}$ such that

$$
\begin{aligned}
& \alpha_{k-1}>a_{2}\left(k c_{k}\right) \sum_{j=1}^{k} j^{2} c_{j}, \\
& \alpha_{i-1}-\alpha_{i}>a_{2}\left(\sum_{l=i}^{k} l c_{l}\right) \sum_{j=1}^{k} j^{2} c_{j}, \quad i=2, \ldots, k-1, \\
& 1-\alpha_{1}>a_{2}\left(\sum_{l=1}^{k} l c_{l}\right) \sum_{j=1}^{k} j^{2} c_{j} .
\end{aligned}
$$

Using these numbers define a function $g$

$$
g\left(x_{0}, x_{1}, \ldots, x_{k-1}\right)=\sum_{j=0}^{k-1} \alpha_{j} x_{j}^{4}, \quad \alpha_{0}=1 .
$$

As before, we need to show that the difference $g-P g$ can be made as large as we wish, away from a compact set. We have:

$$
\begin{aligned}
g\left(r_{n}, r_{n-1}, \ldots, r_{n-k+1}\right)-\operatorname{Pg}\left(r_{n}, r_{n-1}, \ldots, r_{n-k+1}\right) & = \\
= & \sum_{j=0}^{k-1} \alpha_{j} r_{n-j}^{4}-\sum_{j=1}^{k-1} \alpha_{j} r_{n-j+1}^{4}-a_{2}\left[c_{0}+\sum_{l=1}^{k} c_{l}\left(\sum_{d=1}^{l} r_{n-d+1}\right)^{2}\right]^{2} \\
= & \sum_{j=0}^{k-1} \alpha_{j} r_{n-j}^{4}-\sum_{j=1}^{k-1} \alpha_{j} r_{n-j+1}^{4}-a_{2}\left[\sum_{l=1}^{k} c_{l}\left(\sum_{d=1}^{l} r_{n-d+1}\right)^{2}\right]^{2}-\Theta,
\end{aligned}
$$

with $\Theta$ being a polynomial of order 2 . Thus, it is enough to prove that, away from the origin,

$$
\sum_{j=0}^{k-1} \alpha_{j} r_{n-j}^{4}-\sum_{j=1}^{k-1} \alpha_{j} r_{n-j+1}^{4}-a_{2}\left(\sum_{l=1}^{k} c_{l}\left(\sum_{d=1}^{l} r_{n-d+1}\right)^{2}\right)^{2}>0 .
$$

With the help of (8.23)-(8.25), we estimate the left hand side of (8.28) as

$$
\begin{aligned}
& \geq \sum_{j=0}^{k-1} \alpha_{j} r_{n-j}^{4}-\sum_{j=1}^{k-1} \alpha_{j} r_{n-j+1}^{4}-a_{2}\left(\sum_{l=1}^{k} l c_{l} \sum_{d=1}^{l} r_{n-d+1}^{2}\right)^{2} \\
& =\sum_{j=0}^{k-1} \alpha_{j} r_{n-j}^{4}-\sum_{j=1}^{k-1} \alpha_{j} r_{n-j+1}^{4}-a_{2}\left(\sum_{d=1}^{k} r_{n-d+1}^{2} \sum_{l=d}^{k} l c_{l}\right)^{2}
\end{aligned}
$$




$$
\begin{aligned}
& \geq \sum_{j=0}^{k-1} \alpha_{j} r_{n-j}^{4}-\sum_{j=1}^{k-1} \alpha_{j} r_{n-j+1}^{4}-a_{2}\left(\sum_{d=1}^{k} \sum_{l=d}^{k} l c_{l}\right) \sum_{d=1}^{k} r_{n-d+1}^{4} \sum_{l=d}^{k} l c_{l} \\
& =\sum_{j=0}^{k-1} \alpha_{j} r_{n-j}^{4}-\sum_{j=1}^{k-1} \alpha_{j} r_{n-j+1}^{4}-a_{2}\left(\sum_{i=1}^{k} i^{2} c_{i}\right) \sum_{j=0}^{k-1} r_{n-j}^{4} \sum_{l=j+1}^{k} l c_{l} \\
& =\sum_{j=0}^{k-2} r_{n-j}^{4}\left(\alpha_{j}-\alpha_{j+1}-a_{2}\left(\sum_{i=1}^{k} i^{2} c_{i}\right) \sum_{l=j+1}^{k} l c_{l}\right)+ \\
& \quad+r_{n-k+1}^{4}\left(\alpha_{k-1}-a_{2}\left(k c_{k}\right) \sum_{i=1}^{k} i^{2} c_{i}\right)>0 .
\end{aligned}
$$

This proves (8.28), and so (8.22) is a sufficient condition for the existence of a finite 4 th moment for the HARCH(k) models.

\section{General moments of HARCH(k)}

Let $m \geq 2$. As always, if the $(2 \mathrm{~m})$ th moments are finite, there is a system of equations these moments must satisfy. Define, under the assumption of stationarity,

$$
\begin{aligned}
& M_{0}=\mathrm{E}\left(r_{n}^{2 m}\right) \\
& n_{i_{0}, i_{1}, \ldots, i_{k-1}}=\mathrm{E}\left(r_{n}^{2 i_{0}} r_{n-1}^{i_{1}} \ldots r_{n-k+1}^{i_{k-1}}\right), \\
& 1 \leq i_{0} \leq m-1, \quad 0 \leq i_{j} \leq 2 m-2, \quad j=1, \ldots, k-1, \\
& 2 i_{0}+i_{1}+\ldots+i_{k-1}=2 m .
\end{aligned}
$$

If the process has a finite $(2 \mathrm{~m})$ th moment, then we have

$$
\begin{aligned}
M_{0} & =\mathrm{E}\left(r_{n}^{2 m}\right)=\mathrm{E}\left(\varepsilon_{n}^{2 m}\right) \mathrm{E}\left(\sigma_{n}^{2 m}\right)=a_{m} \mathrm{E}\left(\sigma_{n}^{2 m}\right) \\
& \left.=a_{m} \mathrm{E}\left[\left(c_{0}+\sum_{j=1}^{k} c_{j}\left(\sum_{d=1}^{j} r_{n-d}\right)^{2}\right)^{m}\right] a_{m} \vartheta_{0, m}+a_{m} \mathrm{E}\left[\sum_{j=1}^{k} c_{j}\left(\sum_{d=1}^{j} r_{n-d}\right)^{2}\right)^{m}\right],
\end{aligned}
$$

where $\vartheta_{0, m}$ involves only moments of orders less than $2 m$. We further have

$$
\mathrm{E}\left[\left(\sum_{j=1}^{k} c_{j}\left(\sum_{d=1}^{j} r_{n-d}\right)^{2}\right)^{m}\right]=\sum_{p_{1}, \ldots, p_{k}}^{m} \frac{m !}{p_{1} ! \ldots p_{k} !} \prod_{i=1}^{k} c_{i}^{p_{i}} \mathrm{E}\left[\prod_{j=1}^{k}\left(\sum_{d=1}^{j} r_{n-d}\right)^{2 p_{j}}\right] .
$$


with the following relations

$$
0 \leq p_{i} \leq m, \quad \text { and } \sum_{i=1}^{k} p_{i}=m, \quad i=1, \ldots, k
$$

Concentrating on the expectation in (9.4), we further get

$$
\begin{aligned}
\mathrm{E}\left[\prod_{j=1}^{k}\left(\sum_{d=1}^{j} r_{n-d}\right)^{2 p_{j}}\right] & = \\
\quad= & \sum_{l \in \mathcal{L}\left(p_{1}, \ldots, p_{k}\right)} \prod_{i=0}^{k-1}\left(\begin{array}{c}
2\left(p_{k-i}+\ldots+p_{k}\right)-\left(l_{k-i+1}+\ldots+l_{k}\right) \\
l_{k-i}
\end{array}\right) \cdot \mathrm{E}\left[\prod_{j=1}^{k} r_{n-j}^{l_{j}}\right],
\end{aligned}
$$

where for a vector $\underline{p}=\left(p_{1}, \ldots, p_{k}\right)$,

$$
\begin{aligned}
& \mathcal{L}(\underline{p})=\mathcal{L}\left(p_{1}, \ldots, p_{k}\right) \\
&=\left\{\underline{l}=\left(l_{1}, \ldots, l_{k}\right) \mid 0 \leq l_{k} \leq 2 p_{k}, 0 \leq l_{k-1}+l_{k} \leq 2\left(p_{k-1}+p_{k}\right), \ldots,\right. \\
&\left.0 \leq l_{2}+\ldots+l_{k} \leq 2\left(p_{2}+\ldots+p_{k}\right), l_{1}+l_{2}+\ldots+l_{k}=2\left(p_{1}+p_{2}+\ldots+p_{k}\right)\right\}
\end{aligned}
$$

Define a function $b: \mathbb{R}^{k} \longrightarrow\{1, \ldots, k\}$ by

$$
b\left(x_{1}, \ldots, x_{k}\right)=\min \left\{j, x_{j} \neq 0\right\} .
$$

Observe that for every $\underline{l} \in \mathcal{L}(\underline{p})$ we have

$$
b(\underline{l}) \leq b(\underline{p}) .
$$

Indeed, if $b(\underline{l})>j$, then $l_{1}=l_{2}=\ldots=l_{j}=0$, and so we have

$$
2\left(p_{1}+\ldots+p_{k}\right)=l_{1}+\ldots+l_{k}=l_{j+1}+\ldots+l_{k} \leq 2\left(p_{j+1}+\ldots+p_{k}\right),
$$

implying that $p_{1}=p_{2}=\ldots=p_{j}=0$, and so $b(\underline{p})>j$. It is clear that for every $\underline{l} \in \mathcal{L}(\underline{p})$ such that $l_{b(\underline{l})}$ is odd, we have

$$
\mathrm{E}\left[\prod_{j=1}^{k} r_{n-j}^{l_{j}}\right]=0
$$

Therefore, by (9.9), we have

$$
\mathrm{E}\left[\prod_{j=1}^{k}\left(\sum_{d=1}^{j} r_{n-d}\right)^{2 p_{j}}\right]=\sum_{i=1}^{b(\underline{p})}
$$




$$
\left[\sum_{\underline{l} \in \mathcal{L}(\underline{p})} \prod_{j=0}^{k-1}\left(\begin{array}{c}
2\left(p_{k-j}+\ldots+p_{k}\right)-\left(l_{k-j+1}+\ldots+l_{k}\right) \\
l_{k-j}
\end{array}\right) \cdot n_{l_{1} / 2, l_{2}, \ldots, l_{k}}+i M_{0}\right]
$$

with the following condition on the indices of the second summation

$$
b(\underline{l})=i, \quad l_{i} \text { even, } \quad l_{i} \neq 2 m .
$$

Substituting (9.10) into (9.4), we obtain

$$
\begin{aligned}
& \mathrm{E}\left[\left(\sum_{j=1}^{k} c_{j}\left(\sum_{d=1}^{j} r_{n-d}\right)^{2}\right)^{m}\right]=\sum_{i=1}^{k} \sum_{p_{i}, \ldots, p_{k}} \frac{l !}{p_{i} ! \ldots p_{k} !} \prod_{j=i}^{k} c_{j}^{p_{j}} \cdot \\
& \cdot \sum_{d=1}^{i}\left[\sum_{\underline{l} \in \mathcal{L}(\underline{p})} \prod_{j=0}^{k-d}\left(\begin{array}{c}
2\left(p_{k-j}+\ldots+p_{k}\right)-\left(l_{k-j+1}+\ldots+l_{k}\right) \\
l_{k-j}
\end{array}\right) \cdot n_{l_{d} / 2, l_{d+1}, l_{k}, 0, \ldots, 0}+d M_{0}\right],
\end{aligned}
$$

where the indices $\underline{p}$ and $\underline{l}$ follow (9.5) and(9.11) respectively, with $p_{1}=\ldots=p_{i-1}=0$. That is, we have:

$$
\begin{gathered}
\mathrm{E}\left[\left(\sum_{j=1}^{k} c_{j}\left(\sum_{d=1}^{j} r_{n-d}\right)^{2}\right)^{m}\right]=M_{0} \sum_{j=1}^{k}\left(\sum_{i=j}^{k} c_{j}\right)^{m}+\sum_{i=1}^{k} \sum_{p_{i}, \ldots, p_{k}} \frac{l !}{p_{i} ! \ldots p_{k} !} \prod_{j=i}^{k} c_{j}^{p_{j}} . \\
\cdot \sum_{d=1}^{i} \sum_{\underline{l} \in \mathcal{L}(\underline{p})} n_{l_{d} / 2, l_{d+1}, \ldots, l_{k}, 0, \ldots, 0} \prod_{j=0}^{k-d}\left(\begin{array}{c}
2\left(p_{k-j}+\ldots+p_{k}\right)-\left(l_{k-j+1}+\ldots+l_{k}\right) \\
l_{k-j}
\end{array}\right),
\end{gathered}
$$

where the subscript of $n$ above is of length $k$. Therefore, we obtain the following equation

$$
\begin{aligned}
& M_{0}=M_{0} a_{m} \sum_{j=1}^{k}\left(\sum_{i=j}^{k} c_{j}\right)^{m}+a_{m} \sum_{i=1}^{k} \sum_{p_{i}, \ldots, p_{k}} \frac{l !}{p_{i} ! \ldots p_{k} !} \prod_{j=i}^{k} c_{j}^{p_{j}} \cdot \\
& \cdot \sum_{d=1}^{i} \sum_{\underline{l} \in \mathcal{L}(\underline{p})} n_{l_{d} / 2, l_{d+1}, \ldots, l_{k}, 0, \ldots, 0} \prod_{j=0}^{k-d}\left(\begin{array}{c}
2\left(p_{k-j}+\ldots+p_{k}\right)-\left(l_{k-j+1}+\ldots+l_{k}\right) \\
l_{k-j}
\end{array}\right)+a_{m} \vartheta_{0, m} .
\end{aligned}
$$

In a similar manner, it is possible to obtain a system of equations for $n_{i_{0}, i_{1}, \ldots, i_{k-1}}$. This gives us a system of

$$
\sum_{i=0}^{m-1}\left(\begin{array}{c}
2 i+k-2 \\
k-2
\end{array}\right)
$$

linear equations with as many unknowns. We call this system $\varrho_{m}$.

Combining the ideas we used in analyzing the cases of $k=2$ and $m=1$ (Section 6.2), we obtain in the same way, the following theorem. 
Theorem 2 A HARCH $(k)$ model has a stationary version with a finite $(2 m)$ th moment if and only if, for every $j=1, \ldots, m$, the system $\varrho_{j}$ has a positive solution. In this case, $\varrho_{k}$ has a unique solution, given by (9.1) and (9.2).

\subsection{An explicit necessary condition}

Since by Theorem 2, we have:

$$
n_{i_{0}, i_{1}, \ldots, i_{k}} \geq 0 \text { for all } i_{0}, i_{1}, \ldots, i_{k}
$$

it follows from (9.12) that

$$
M_{0}>a_{m} M_{0} \sum_{j=1}^{k}\left(\sum_{i=j}^{k} c_{j}\right)^{m}
$$

and so

$$
\sum_{j=1}^{k}\left(\sum_{i=j}^{k} c_{j}\right)^{m}<\frac{1}{a_{m}}
$$

is a necessary condition for the existence of a finite $(2 m)$ th moment.

\subsection{An explicit sufficient condition}

We claim that if

$$
\left(\sum_{j=1}^{k} j^{2} c_{j}\right)^{m}<\frac{1}{a_{m}}
$$

then the HARCH(k) model has a finite $(2 m)$ th moment.

The proof proceeds exactly like in the case $m=2$ (Section 8.3). We take

$$
g\left(x_{0}, x_{1}, \ldots, x_{k-1}\right)=\sum_{j=0}^{k-1} \alpha_{j} x_{j}^{2 m}, \quad \alpha_{0}=1
$$

and note that under (9.14) we may choose $\alpha_{1}, \ldots, \alpha_{k-1}$ in such a way that

$$
\alpha_{k-1}>a_{m}\left(k c_{k}\right)\left(\sum_{j=1}^{k} j^{2} c_{j}\right)^{m-1}
$$




$$
\begin{aligned}
& \alpha_{i-1}-\alpha_{i}>a_{m}\left(\sum_{l=i}^{k} l c_{l}\right)\left(\sum_{j=1}^{k} j^{2} c_{j}\right)^{m-1}, \quad i=2, \ldots, k-1, \\
& 1-\alpha_{1}>a_{m}\left(\sum_{l=1}^{k} l c_{l}\right)\left(\sum_{j=1}^{k} j^{2} c_{j}\right)^{m-1} .
\end{aligned}
$$

Then proceed exactly as in Section 8.3.

\section{Summary}

We give in this paper a necessary and sufficient condition for the existence of moments of a general stationary HARCH $(\mathrm{k})$ process using a Markov chain approach. Our interest in the tail behavior of this process leads us to study the convergence of the higher order moments of HARCH processes. We give a theorem that governs the stationarity with finite moments and we prove that the condition is both necessary and sufficient. The condition is in terms of the existence of non-negative solution of a system of linear equations. Unfortunately, the general condition we give is not explicit. For every $\mathrm{HARCH}(\mathrm{k})$ process one has explicit necessary and sufficient conditions for finiteness of the second moment. In the case of the $\mathrm{HARCH}(2)$ process, we can give the explicit necessary and sufficient condition for the existence of the 4 th moment. In all other cases, we give explicit necessary conditions and explicit sufficient conditions which will allow one to study the tail behavior of $\mathrm{HARCH}$ processes.

\section{References}

Billingsley P., 1968, Convergence of probability measures, Wiley, New York.

Bollerslev T., 1986, Generalized autoregressive conditional heteroskedasticity, Journal of Econometrics, 31, 307-327.

Dacorogna M. M., Müller U. A., Nagler R. J., Olsen R. B., and Pictet O. V., 1993, A geographical model for the daily and weekly seasonal volatility in the FX market, Journal of International Money and Finance, 12(4), 413-438.

Engle R. F., 1982, Autoregressive conditional heteroskedasticity with estimates of the variance of $U$. $K$. inflation, Econometrica, 50, 987-1008.

Guillaume D. M., Dacorogna M. M., Davé R. D., Müller U. A., Olsen R. B., and Pictet O. V., 1994, From the bird's eye to the microscope: A survey of new stylized facts of the intra-daily foreign exchange markets, Internal document DMG.1994-04-06, Olsen \& Associates, Seefeldstrasse 233, 8008 Zürich, Switzerland.

Meyn S. P. and Tweedie R. L., 1990, Markov Chains and Stochastic Stability, Springer Verlag, Heidelberg. 
Müller U. A., Dacorogna M. M., Davé R. D., Olsen R. B., Pictet O. V., and von Weizsäcker J. E., 1995, Volatilities of different time resolutions - analyzing the dynamics of market components, forthcoming in the Journal of Empirical Finance, UAM.1995-01-12, Olsen \& Associates, Seefeldstrasse 233, 8008 Zürich, Switzerland.

Paul Embrechts

\section{Department of Mathematics}

ETH Zentrum

CH-8092 Zürich

Switzerland

telephone 41-1-632-34-19, e-mail: embrecht s@math . ethz . ch

Gennady Samorodnitsky

School of Operations Research and Industrial Engineering

Cornell University

Ithaca, NY 14853

U.S.A.

telephone 1-607-255-9141, e-mail: gennady@orie. cornell . edu

Michel M. Dacorogna

Olsen \& Associates

Research Institute for Applied Economics

Seefeldstrasse 233

CH-8008 Zürich

Switzerland

telephone 41-1-386-48-48, e-mail: daco@olsen . ch

Ulrich A. Müller

Olsen \& Associates

Research Institute for Applied Economics

Seefeldstrasse 233

CH-8008 Zürich

Switzerland

telephone 41-1-386-48-48, e-mail: ulrichm@olsen. ch 